\title{
Complex and Kähler structures on hom-Lie algebras
}

\author{
E. Peyghan*(D), L. Nourmohammadifar (D) \\ Department of Mathematics, Faculty of Science, Arak University, Arak, 38156-8-8349, Iran.
}

\begin{abstract}
(Almost) Complex and Hermitian structures on hom-Lie algebras are introduced and some examples of these structures are presented. We study the complexification of hom-Lie algebras. Also, the notion of Kähler hom-Lie algebras is introduced and then using a Kähler hom-Lie algebra, we present a phase space. Finally, we describe all two-dimension non-abelian Kähler hom-Lie algebra and also it is shown that there does not exist a nonabelian Kähler proper hom-Lie algebra of dimension two.
\end{abstract}

Mathematics Subject Classification (2010). 17A30, 17D25, 53C15, 53D05

Keywords. proper hom-Lie algebra, almost Hermitian structure, hom-Levi-Civita product, Kähler hom-Lie algebra, phase space

\section{Introduction}

The notion of hom-Lie algebras was introduced by Hartwig, Larsson, and Silvestrov in the study of $\sigma$-deformations of the Witt and Virasoro algebras in [5]. Indeed, some $q$-deformations of the Witt and the Virasoro algebras have the structure of a hom-Lie algebra $[5,6]$. Based on the close relation between the discrete, deformed vector fields and differential calculus, this algebraic structure plays an important role in research fields $[3,5,8-12,15]$.

Differential-geometric structures play an important role in the study of complex geometry. After Kodaira, Kähler structures became central in the study of deformation theory and the classification problems. Recall that an almost complex structure $J$ on a manifold $M$ is a linear complex structure (that is, a linear map which squares to -1) on each tangent space of the manifold, which varies smoothly on the manifold. A complex structure is essentially an almost complex structure with an integrability condition, and this condition yields an unitary structure $(U(n)$ structure) on the manifold. An almost Hermitian structure is a pair $(J, g)$ of an almost complex structure $J$ and a pseudo-Riemannian metric $g$ such that $g(\cdot, \cdot)=g(J \cdot, J \cdot)$. A manifold $M$ is called almost Hermitian manifold if it is endowed with an almost Hermitian structure $(J, g)$. An almost Hermitian manifold $(M, J, g)$ is called Kähler, if its Levi-Civita connection $\nabla$ satisfies $\nabla J=0$. When the manifold is a Lie group $G$, the metric and the complex structure are considered left-invariant hence they are both determined by their restrictions to the Lie algebra $\mathfrak{g}$ of $G$. Further, the existence

\footnotetext{
*Corresponding Author.

Email addresses: e-peyghan@araku.ac.ir (E. Peyghan), l.nourmohammadi@gmail.com (L. Nourmohammadifar)

Received: 28.11.2018; Accepted: 30.06.2019
} 
of a left invariant Kähler structure on $G$ reduces to the existence of a symplectic form on the Lie algebra $\mathfrak{g}$. In this situation, $\left(\mathfrak{g}, g_{e}, J_{e}\right)$ is called a Kähler Lie algebra. Complex, complex product and contact structures on Lie algebras have been studied widely $[1,2,4]$.

The aim of this paper is to develop theories that parallel to manifolds for hom-Lie algebras. It is known that hom-Lie algebras as generalizations of Lie algebras have close relationships with statistical mechanics and mathematical physics. Also, Kähler structures play applicable and important roles in mathematical physics. So, introducing and studying of these structures on hom-Lie algebras can be useful in mathematical physics. On the other hand, recently some researchers introduced hom-Lie groups as generalizations of Lie groups and they studied the relationships between hom-Lie groups and hom-Lie algebras $[7,16]$. Indeed, it looks natural that hom-Lie group-hom-Lie algebra correspondence allows one to study the hom-Lie groups-hom Lie algebra, which are geometric objects, in terms of hom-Lie algebras, which are linear objects. So, we can study many geometric concepts such as complex, Hermitian and Kähler structures on hom-Lie groups by using the corresponding hom-Lie algebras.

The structure of this paper is organized as follows: In Section 2, we recall the definition of hom-Lie algebra and pseudo-Riemannian hom-algebra and hom-Levi-Civita product. Also we present an example of a symplectic hom-Lie algebra. In Section 3, we introduce complex and Hermitian structures on hom-Lie algebras. Also, we present an example of an almost Hermitian hom-Lie algebra. Then we study the complexification of a hom-Lie algebra. In Section 4, we define the phase space of a hom-Lie algebra and then using an involutive hom-left symmetric algebra we construct a phase space. Also, we present a complex structure on this phase space. In Section 5, we introduce Kähler home Lie algebras and we present an example of them. Then we study some of their properties and we show that using these structures we can construct phase spaces. Also, we determine all complex hom-Lie algebra of dimension 2. Finally, we show that there do not exist non-abelian Hermitian and Kähler proper hom-Lie algebras of dimension 2. In particular, we classify non-abelian Hermitian and Kähler hom-Lie algebras of dimension 2.

In this paper, we work over the real field $\mathbb{R}$ and the complex field $\mathbb{C}$.

\section{Preliminaries}

In this section, we recall some basic definitions on hom-category.

Definition 2.1 ([12]). A hom-algebra is a triple $(V, \cdot, \phi)$ consisting of a linear space $V$, a bilinear map (product) $: V \times V \rightarrow V$ and an algebra morphism $\phi: V \rightarrow V$.

Let $(V, \cdot, \phi)$ be a hom-algebra. Then for any $u \in V$, we consider maps $L_{u}, R_{u}: V \rightarrow V$ as the left and the right multiplication by $u$ given by $L_{u}(v)=u \cdot v$ and $R_{u}(v)=v \cdot u$, respectively. The commutator on $V$ is given by $[u, v]=u \cdot v-v \cdot u$.

Definition $2.2([9])$. A hom-Lie algebra is a triple $\left(\mathfrak{g},[\cdot, \cdot], \phi_{\mathfrak{g}}\right)$ consisting of a linear space $\mathfrak{g}$, a bilinear map (bracket) $[\cdot, \cdot]: \mathfrak{g} \times \mathfrak{g} \rightarrow \mathfrak{g}$ and an algebra morphism $\phi_{\mathfrak{g}}: \mathfrak{g} \rightarrow \mathfrak{g}$ satisfying the following hom-Jacobi identity:

$$
[u, v]=-[v, u], \quad \circlearrowleft_{u, v, w}\left[\phi_{\mathfrak{g}}(u),[v, w]\right]=0,
$$

for any $u, v, w \in \mathfrak{g}$, where $\circlearrowleft$ is the symbol of cyclic sum.

The hom-Lie algebra $\left(\mathfrak{g},[\cdot, \cdot], \phi_{\mathfrak{g}}\right)$ is called regular hom-Lie algebra (respectively, involutive hom-Lie algebra), if $\phi_{\mathfrak{g}}$ is non-degenerate (respectively, satisfies $\phi_{\mathfrak{g}}{ }^{2}=I d_{\mathfrak{g}}$ ). It is known that a Lie algebra $(\mathfrak{g},[\cdot, \cdot])$ with $\phi_{\mathfrak{g}}=I d_{\mathfrak{g}}$ is a hom-Lie algebra. We call $\left(\mathfrak{g},[\cdot, \cdot], \phi_{\mathfrak{g}}\right)$ proper hom-Lie algebra if $\phi_{\mathfrak{g}} \neq I d_{\mathfrak{g}}$.

Also, a subspace $\mathfrak{h} \subset \mathfrak{g}$ is called a hom-Lie subalgebra of $\mathfrak{g}$ if $\phi_{\mathfrak{g}}(\mathfrak{h}) \subset \mathfrak{h}$ and $[u, v] \in \mathfrak{h}$, for any $u, v \in \mathfrak{h}$. 
Definition 2.3 ([12]). For any finite-dimensional hom-Lie algebra $\left(\mathfrak{g},[\cdot, \cdot], \phi_{\mathfrak{g}}\right)$, we can consider a bilinear symmetric non-degenerate form $\langle\cdot, \cdot\rangle$ such that for any $u, v \in \mathfrak{g}$ the following equation is satisfied

$$
\left\langle\phi_{\mathfrak{g}}(u), \phi_{\mathfrak{g}}(v)\right\rangle=\langle u, v\rangle .
$$

In this case, we say that $\mathfrak{g}$ admits a pseudo-Riemannian metric $\langle\cdot, \cdot\rangle$ and $\left(\mathfrak{g},[\cdot, \cdot], \phi_{\mathfrak{g}},\langle\cdot, \cdot\rangle\right)$ is called pseudo-Riemannian hom-Lie algebra. Also, if $\phi_{\mathfrak{g}}^{2}=I d_{\mathfrak{g}}$, then we have

$$
\left\langle\phi_{\mathfrak{g}}(u), v\right\rangle=\left\langle u, \phi_{\mathfrak{g}}(v)\right\rangle .
$$

It can be verified that on a pseudo-Riemannian regular hom-Lie algebra $\left(\mathfrak{g},[\cdot, \cdot], \phi_{\mathfrak{g}},\langle\cdot, \cdot\rangle\right)$, there exists a unique product · which is given by Koszul's formula

$$
2\left\langle u \cdot v, \phi_{\mathfrak{g}}(w)\right\rangle=\left\langle[u, v], \phi_{\mathfrak{g}}(w)\right\rangle+\left\langle[w, v], \phi_{\mathfrak{g}}(u)\right\rangle+\left\langle[w, u], \phi_{\mathfrak{g}}(v)\right\rangle,
$$

which satisfies

$$
\begin{aligned}
{[u, v] } & =u \cdot v-v \cdot u, \\
\left\langle u \cdot v, \phi_{\mathfrak{g}}(w)\right\rangle & =-\left\langle\phi_{\mathfrak{g}}(v), u \cdot w\right\rangle .
\end{aligned}
$$

This product is called the hom-Levi-Civita product.

\section{3. (Almost) Complex and Hermitian structures on hom-Lie algebras}

In this section, we introduce (almost) complex and Hermitian structures on hom-Lie algebras. Also, we introduce the complexification of hom-Lie algebras.

\subsection{Hermitian structures on hom-Lie algebras}

Definition 3.1. An almost complex structure on an involutive hom-Lie algebra $\left(\mathfrak{g},[\cdot, \cdot], \phi_{\mathfrak{g}}\right)$, is an isomorphism $J: \mathfrak{g} \rightarrow \mathfrak{g}$ that satisfies $J^{2}=-I d_{\mathfrak{g}}$ and $\phi_{\mathfrak{g}} \circ J=J \circ \phi_{\mathfrak{g}}$.

An immediate corollary of the above definition is that $\left(\phi_{\mathfrak{g}} \circ J\right)^{2}=-I d_{\mathfrak{g}}$. We denote an almost complex hom-Lie algebra by $\left(\mathfrak{g},[\cdot, \cdot], \phi_{\mathfrak{g}}, J\right)$. If the $n$-dimensional hom-Lie algebra $\mathfrak{g}$ admits an almost complex structure $J$, then

$$
(\operatorname{det}(J))^{2}=\operatorname{det}\left(J^{2}\right)=\operatorname{det}\left(-I d_{\mathfrak{g}}\right)=(-1)^{n},
$$

which implies that $n$ is even.

The Nijenhuis torsion $N_{\phi_{\mathfrak{g}} \circ J}$ (or simply $N$ ) of $\phi_{\mathfrak{g}} \circ J$ is defined by

$$
N(u, v)=\left[\left(\phi_{\mathfrak{g}} \circ J\right) u,\left(\phi_{\mathfrak{g}} \circ J\right) v\right]-\phi_{\mathfrak{g}} \circ J\left[\left(\phi_{\mathfrak{g}} \circ J\right) u, v\right]-\phi_{\mathfrak{g}} \circ J\left[u,\left(\phi_{\mathfrak{g}} \circ J\right) v\right]-[u, v],
$$

for all $u, v \in \mathfrak{g}$. We will say that $\phi_{\mathfrak{g}} \circ J$ is integrable if $N=0$ and hence we will call $J$ a complex structure on $\mathfrak{g}$.

Definition 3.2. An almost Hermitian structure on the hom-Lie algebra $\left(\mathfrak{g},[\cdot, \cdot], \phi_{\mathfrak{g}}\right)$ is a pair $(J,\langle\cdot, \cdot\rangle)$ of an almost complex structure $J$ and a pseudo-Riemannian metric $\langle\cdot, \cdot\rangle$ on $\mathfrak{g}$ such that

$$
\left\langle\left(\phi_{\mathfrak{g}} \circ J\right) u,\left(\phi_{\mathfrak{g}} \circ J\right) v\right\rangle=\langle u, v\rangle, \quad \forall u, v \in \mathfrak{g},
$$

and if $\phi_{\mathfrak{g}} \circ J$ is integrable, then the pair $(J,\langle\cdot, \cdot\rangle)$ is called Hermitian structure on $\mathfrak{g}$. In this case, $\left(\mathfrak{g},[\cdot, \cdot], \phi_{\mathfrak{g}}, J,\langle\cdot, \cdot\rangle\right)$ is called Hermitian hom-Lie algebra.

According to the above definition, we present an example of a 4-dimensional space.

Example 3.3. We consider a 4 -dimensional hom-Lie algebra $\left(\mathfrak{g},[\cdot, \cdot], \phi_{\mathfrak{g}}\right)$ with an arbitrary basis $\left\{e_{1}, e_{2}, e_{3}, e_{4}\right\}$ such that bracket $[\cdot, \cdot]$ and linear map $\phi_{\mathfrak{g}}$ on $\mathfrak{g}$ are defined by

$$
\left[e_{1}, e_{3}\right]=a e_{1}+a e_{2}, \quad\left[e_{2}, e_{4}\right]=a e_{1}+a e_{2}, \quad\left[e_{3}, e_{4}\right]=-a e_{3}+a e_{4},
$$

and

$$
\phi_{\mathfrak{g}}\left(e_{1}\right)=e_{2}, \quad \phi_{\mathfrak{g}}\left(e_{2}\right)=e_{1}, \quad \phi_{\mathfrak{g}}\left(e_{3}\right)=e_{4}, \quad \phi_{\mathfrak{g}}\left(e_{4}\right)=e_{3} .
$$


The above bracket is not a Lie bracket on $\mathfrak{g}$ if $a \neq 0$. Now we consider the metric $\langle\cdot, \cdot\rangle$ of $\mathfrak{g}$ as follows:

$$
\left[\begin{array}{llll}
1 & 0 & 0 & 0 \\
0 & 1 & 0 & 0 \\
0 & 0 & 1 & 0 \\
0 & 0 & 0 & 1
\end{array}\right] .
$$

It can be checked easily that $\left\langle\phi_{\mathfrak{g}}\left(e_{i}\right), e_{j}\right\rangle=\left\langle e_{i}, \phi_{\mathfrak{g}}\left(e_{j}\right)\right\rangle$, for all $i, j=1,2,3,4$. If isomorphism $J$ is determined as

$$
J\left(e_{1}\right)=e_{4}, \quad J\left(e_{2}\right)=e_{3}, \quad J\left(e_{3}\right)=-e_{2}, \quad J\left(e_{4}\right)=-e_{1},
$$

then we have

$$
\phi_{\mathfrak{g}}^{2}\left(e_{i}\right)=-J^{2}\left(e_{i}\right)=e_{i}, \quad i=1,2,3,4 .
$$

A simple calculation shows that

$$
\begin{aligned}
& \left(J \circ \phi_{\mathfrak{g}}\right) e_{1}=e_{3}=\left(\phi_{\mathfrak{g}} \circ J\right) e_{1}, \quad\left(J \circ \phi_{\mathfrak{g}}\right) e_{2}=e_{4}=\left(\phi_{\mathfrak{g}} \circ J\right) e_{2}, \\
& \left(J \circ \phi_{\mathfrak{g}}\right) e_{3}=-e_{1}=\left(\phi_{\mathfrak{g}} \circ J\right) e_{3}, \quad\left(J \circ \phi_{\mathfrak{g}}\right) e_{4}=-e_{2}=\left(\phi_{\mathfrak{g}} \circ J\right) e_{4} .
\end{aligned}
$$

Thus $J$ is an almost complex structure on $\left(g,[\cdot, \cdot], \phi_{\mathfrak{g}}\right)$. Also, we see that

$$
\left\langle\left(\phi_{\mathfrak{g}} \circ J\right)\left(e_{i}\right),\left(\phi_{\mathfrak{g}} \circ J\right)\left(e_{j}\right)\right\rangle=\left\langle e_{i}, e_{j}\right\rangle, \quad \forall i, j=1,2,3,4 .
$$

Hence $\left(\mathfrak{g},[\cdot, \cdot], \phi_{\mathfrak{g}},\langle\cdot, \cdot\rangle, J\right)$ is an almost Hermitian hom-Lie algebra. It is easy to see that

$$
N\left(e_{1}, e_{2}\right)=-a e_{1}+a e_{2} \neq 0 .
$$

So, $J$ is not integrable and consequently $\left(\mathfrak{g},[\cdot, \cdot], \phi_{\mathfrak{g}},\langle\cdot, \cdot\rangle, J\right)$ is not a Hermitian hom-Lie algebra.

\subsection{Complexification of hom-Lie algebras}

We denote the complexification of a hom-Lie algebra $\mathfrak{g}$ by $\mathfrak{g}^{\mathbb{C}}$ and we define it as follows

$$
\mathfrak{g}^{\mathbb{C}}=\{u+i v \mid u, v \in \mathfrak{g}\} .
$$

If $J$ is an almost complex structure on $\mathfrak{g}$, we can extend $J$ and $\phi_{\mathfrak{g}}$ to $\mathfrak{g}^{\mathbb{C}}$ as follows

$$
J(u+i v)=J u+i J v, \quad \phi_{\mathfrak{g}}(u+i v)=\phi_{\mathfrak{g}}(u)+i \phi_{\mathfrak{g}}(v) .
$$

Obviously $J^{2}=-\phi_{\mathfrak{g}}^{2}=\left(\phi_{\mathfrak{g}} \circ J\right)^{2}=-I d_{\mathfrak{g}^{\mathbb{C}}}$ and $\phi_{\mathfrak{g}} \circ J=J \circ \phi_{\mathfrak{g}}$ on $\mathfrak{g}^{\mathbb{C}}$. Now, we consider

$$
\begin{aligned}
& \mathfrak{g}^{1,0}=\left\{w \in \mathfrak{g}^{\mathbb{C}} \mid\left(\phi_{\mathfrak{g}} \circ J\right) w=i w\right\}, \\
& \mathfrak{g}^{0,1}=\left\{w \in \mathfrak{g}^{\mathbb{C}} \mid\left(\phi_{\mathfrak{g}} \circ J\right) w=-i w\right\} .
\end{aligned}
$$

Proposition 3.4. Let $\mathfrak{g}$ be a hom-Lie algebra with an almost complex structure J. Then

i) $\mathfrak{g}^{1,0}=\left\{u-i\left(\phi_{\mathfrak{g}} \circ J\right) u \mid u \in \mathfrak{g}\right\}$ and $\mathfrak{g}^{0,1}=\left\{u+i\left(\phi_{\mathfrak{g}} \circ J\right) u \mid u \in \mathfrak{g}\right\}$,

ii) $\mathfrak{g}^{\mathbb{C}}=\mathfrak{g}^{1,0} \oplus \mathfrak{g}^{0,1}$,

iii) $\overline{\mathfrak{g}^{1,0}}=\mathfrak{g}^{0,1}$.

Proof. Let $w=u+i v \in \mathfrak{g}^{\mathbb{C}}$. Then $\left(\phi_{\mathfrak{g}} \circ J\right) w=i w$ if and only if

$$
\left(\phi_{\mathfrak{g}} \circ J\right) u+v+i\left(\left(\phi_{\mathfrak{g}} \circ J\right) v-u\right)=0,
$$

which gives $v=-\left(\phi_{\mathfrak{g}} \circ J\right) u$. Similarly, we see that $\left(\phi_{\mathfrak{g}} \circ J\right) w=-i w$ if and only if $v=\left(\phi_{\mathfrak{g}} \circ J\right) u$. Thus we have $(i)$. Let $w=u+i v \in \mathfrak{g}^{\mathbb{C}}$. Setting

$$
\begin{aligned}
w_{1} & =\frac{1}{2}\left(u-i\left(\phi_{\mathfrak{g}} \circ J\right) u+i\left(v-i\left(\phi_{\mathfrak{g}} \circ J\right) v\right)\right), \\
w_{2} & =\frac{1}{2}\left(u+i\left(\phi_{\mathfrak{g}} \circ J\right) u+i\left(v+i\left(\phi_{\mathfrak{g}} \circ J\right) v\right)\right),
\end{aligned}
$$


we get $w_{1} \in \mathfrak{g}^{1,0}$ and $w_{2} \in \mathfrak{g}^{0,1}$. Also, it is easy to see that $w_{1}+w_{2}=u+i v=w$. Therefore, $\mathfrak{g}^{\mathbb{C}}=\mathfrak{g}^{1,0}+\mathfrak{g}^{0,1}$. If $w \in \mathfrak{g}^{1,0} \cap \mathfrak{g}^{0,1}$, i.e., $w=u-i\left(\phi_{\mathfrak{g}} \circ J\right) u$ and $w=v+i\left(\phi_{\mathfrak{g}} \circ J\right) v$, then we obtain

$$
u-i\left(\phi_{\mathfrak{g}} \circ J\right) u=v+i\left(\phi_{\mathfrak{g}} \circ J\right) v
$$

and so

$$
v-u+i\left(\left(\phi_{\mathfrak{g}} \circ J\right) v+\left(\phi_{\mathfrak{g}} \circ J\right) u\right)=0,
$$

which gives $u=v$ and $u=-v$. Hence $\mathfrak{g}^{1,0} \cap \mathfrak{g}^{0,1}=0$ and consequently $\mathfrak{g}^{\mathbb{C}}=\mathfrak{g}^{1,0} \oplus \mathfrak{g}^{0,1}$. Thus we have $(i i)$. Let $w=u+i v \in \mathfrak{g}^{1,0}$. Then we have $v=-\left(\phi_{\mathfrak{g}} \circ J\right) u$. Therefore we get

$$
\left(\phi_{\mathfrak{g}} \circ J\right)(\bar{w})=\left(\phi_{\mathfrak{g}} \circ J\right)(u-i v)=-(v+i u)=-i(u-i v)=-i \bar{w}
$$

which implies $\bar{w} \in \mathfrak{g}^{0,1}$. So we have (iii).

Note that with the natural extensions of the Lie bracket of $\mathfrak{g}$ and $\phi_{\mathfrak{g}}$ to $\mathfrak{g}^{\mathbb{C}}$, this complex vector space has the structure of a complex hom-Lie algebra.

Theorem 3.5. Let $J$ be an almost complex structure on a hom-Lie algebra $\left(\mathfrak{g},[\cdot, \cdot], \phi_{\mathfrak{g}}\right)$. Then the following statements are equivalent:

(i) $\mathfrak{g}^{1,0}$ is a hom-Lie subalgebra of $\mathfrak{g}^{\mathbb{C}}$, i.e., $\left[\mathfrak{g}^{1,0}, \mathfrak{g}^{1,0}\right] \subseteq \mathfrak{g}^{1,0}$ and $\phi_{\mathfrak{g}}\left(\mathfrak{g}^{1,0}\right) \subset \mathfrak{g}^{1,0}$,

(ii) $\mathfrak{g}^{0,1}$ is a hom-Lie subalgebra of $\mathfrak{g}^{\mathbb{C}}$, i.e., $\left[\mathfrak{g}^{0,1}, \mathfrak{g}^{0,1}\right] \subseteq \mathfrak{g}^{0,1}$ and $\phi_{\mathfrak{g}}\left(\mathfrak{g}^{0,1}\right) \subset \mathfrak{g}^{0,1}$,

(iii) $J$ is a complex structure on $\mathfrak{g}$.

Proof. Assume (i) holds. Let $\widetilde{z}, \widetilde{w} \in \mathfrak{g}^{0,1}$. Then according to (iii) of Proposition 3.4, there exist $z, w \in \mathfrak{g}^{1,0}$ such that $\widetilde{z}=\bar{z}$ and $\widetilde{w}=\bar{w}$. On the other hand

$$
\begin{aligned}
{[z, w]=} & {\left[u-i\left(\phi_{\mathfrak{g}} \circ J\right) u, v-i\left(\phi_{\mathfrak{g}} \circ J\right) v\right]=[u, v]-\left[\left(\phi_{\mathfrak{g}} \circ J\right) u,\left(\phi_{\mathfrak{g}} \circ J\right) v\right]-i\left(\left[u,\left(\phi_{\mathfrak{g}} \circ J\right) v\right]\right.} \\
& \left.+\left[\left(\phi_{\mathfrak{g}} \circ J\right) u, v\right]\right),
\end{aligned}
$$

for $u, v \in \mathfrak{g}$. Therefore we have

$$
\begin{aligned}
\overline{[z, w]} & =[u, v]-\left[\left(\phi_{\mathfrak{g}} \circ J\right) u,\left(\phi_{\mathfrak{g}} \circ J\right) v\right]+i\left(\left[u,\left(\phi_{\mathfrak{g}} \circ J\right) v\right]+\left[\left(\phi_{\mathfrak{g}} \circ J\right) u, v\right]\right) \\
& =\left[u+i\left(\phi_{\mathfrak{g}} \circ J\right) u, v+i\left(\phi_{\mathfrak{g}} \circ J\right) v\right]=[\bar{z}, \bar{w}] .
\end{aligned}
$$

According to $(i)$, since $[z, w] \in \mathfrak{g}^{1,0}$, then from Proposition 3.4 and the above equation, we conclude $\overline{[z, w]} \in \mathfrak{g}^{0,1}$. Hence $[\widetilde{z}, \widetilde{w}]=[\bar{z}, \bar{w}]=\overline{[z, w]} \in \mathfrak{g}^{0,1}$. Also, since $\phi_{\mathfrak{g}}(z) \in \mathfrak{g}^{1,0}$, then $\phi_{\mathfrak{g}}(\widetilde{z})=\phi_{\mathfrak{g}}(\bar{z})=\overline{\phi_{\mathfrak{g}}(z)} \in \mathfrak{g}^{0,1}$. Moreover $\left[\phi_{\mathfrak{g}}(z), \phi_{\mathfrak{g}}(w)\right]=\phi_{\mathfrak{g}}[z, w]$ implies

$$
\begin{aligned}
{\left[\phi_{\mathfrak{g}}(\widetilde{z}), \phi_{\mathfrak{g}}(\widetilde{w})\right]=} & {\left[\phi_{\mathfrak{g}}(\bar{z}), \phi_{\mathfrak{g}}(\bar{w})\right]=\left[\overline{\phi_{\mathfrak{g}}(z)}, \overline{\phi_{\mathfrak{g}}(w)}\right]=\overline{\left[\phi_{\mathfrak{g}}(z), \phi_{\mathfrak{g}}(w)\right]}=\overline{\phi_{\mathfrak{g}}[z, w]}=\phi_{\mathfrak{g}} \overline{[z, w]} } \\
& =\phi_{\mathfrak{g}}[\bar{z}, \bar{w}]=\phi_{\mathfrak{g}}[\widetilde{z}, \widetilde{w}] .
\end{aligned}
$$

Therefore $(i)$ implies $(i i)$. In the similar way, $(i i)$ implies $(i)$ and so $(i)$ is equivalent to (ii). Now, let $u, v \in \mathfrak{g}$ and $w:=\left[u+i\left(\phi_{\mathfrak{g}} \circ J\right) u, v+i\left(\phi_{\mathfrak{g}} \circ J\right) v\right]$. Then we get

$$
w=[u, v]-\left[\left(\phi_{\mathfrak{g}} \circ J\right) u,\left(\phi_{\mathfrak{g}} \circ J\right) v\right]+i\left(\left[u,\left(\phi_{\mathfrak{g}} \circ J\right) v\right]+\left[\left(\phi_{\mathfrak{g}} \circ J\right) u, v\right]\right) .
$$

The above equation implies

$$
\begin{aligned}
\left(\phi_{\mathfrak{g}} \circ J\right) w= & \left(\phi_{\mathfrak{g}} \circ J\right)[u, v]-\left(\phi_{\mathfrak{g}} \circ J\right)\left[\left(\phi_{\mathfrak{g}} \circ J\right) u,\left(\phi_{\mathfrak{g}} \circ J\right) v\right]+i\left(\phi_{\mathfrak{g}} \circ J\right)\left(\left[u,\left(\phi_{\mathfrak{g}} \circ J\right) v\right]\right. \\
& \left.+\left[\left(\phi_{\mathfrak{g}} \circ J\right) u, v\right]\right)
\end{aligned}
$$

and

$$
i w=i[u, v]-i\left[\left(\phi_{\mathfrak{g}} \circ J\right) u,\left(\phi_{\mathfrak{g}} \circ J\right) v\right]-\left(\left[u,\left(\phi_{\mathfrak{g}} \circ J\right) v\right]+\left[\left(\phi_{\mathfrak{g}} \circ J\right) u, v\right]\right) .
$$

Summing two last equations, we obtain

$$
\left(\phi_{\mathfrak{g}} \circ J\right) w+i w=-\left(\phi_{\mathfrak{g}} \circ J\right) N(u, v)-i\left(\phi_{\mathfrak{g}} \circ J\right) N(u, v) .
$$

Therefore $w \in \mathfrak{g}^{0,1}$, i.e., $\left(\phi_{\mathfrak{g}} \circ J\right) w=-i w$ if and only if $N=0$, which means that (ii) is equivalent to (iii). 
Remark 3.6. Proposition 3.4 and Theorem 3.5 show that the complexification of a real hom-Lie algebra is a hom-Lie algebra over $\mathbb{C}$.

\section{Phase spaces and representations}

Let $V$ be a real vector space equipped with an inner product. For any $u \in V$, we consider $u^{*} \in V^{*}$ given by $u^{*}(v)=\langle u, v\rangle$, for all $v \in V$. The map $u \rightarrow u^{*}$ defines a canonical isomorphism between $V$ and $V^{*}$. In this paper, we denote $u^{*}(v)$ by $\prec u^{*}, v \succ$.

A symplectic hom-Lie algebra is a regular hom-Lie algebra $\left(\mathfrak{g},[\cdot, \cdot], \phi_{\mathfrak{g}}\right)$ endowed with a bilinear skew-symmetric non-degenerate form $\omega$ which is a 2 -hom-cocycle, i.e.

$$
\begin{gathered}
\omega\left([u, v], \phi_{\mathfrak{g}}(w)\right)+\omega\left([w, u], \phi_{\mathfrak{g}}(v)\right)+\omega\left([v, w], \phi_{\mathfrak{g}}(u)\right)=0, \\
\omega\left(\phi_{\mathfrak{g}}(u), \phi_{\mathfrak{g}}(v)\right)=\omega(u, v) .
\end{gathered}
$$

In this case, $\omega$ is called a symplectic structure on $\mathfrak{g}$.

Definition 4.1. Let $\left(V, \cdot, \phi_{V}\right)$ be a hom-algebra and $V^{*}$ be its dual space. If there exists a hom-algebra structure on the direct sum of the underling vector space $\left(V, \phi_{V}\right)$ and $\left(V^{*}, \phi_{V^{*}}^{*}\right)\left(\phi_{V^{*}}^{*}\right.$ is the transpose of the endomorphism $\left.\phi_{V}\right)$ such that $\left(V, \phi_{V}\right)$ and $\left(V^{*}, \phi_{V^{*}}^{*}\right)$ are sub-hom-algebras and the natural skew-symmetric bilinear form $\omega$ on $V \oplus V^{*}$ given by

$$
\omega\left(u+a^{*}, v+b^{*}\right)=\prec b^{*}, u \succ-\prec a^{*}, v \succ,
$$

is a symplectic form for any $u, v \in V$ and $a^{*}, b^{*} \in V^{*}$, then $\left(V \oplus V^{*}, \omega\right)$ is called phase space of $V$.

Definition 4.2. Let $\left(\mathfrak{g},[.,],. \phi_{\mathfrak{g}}\right)$ be a hom-Lie algebra and $\mathfrak{g}^{*}$ be its dual space. A phase space of $\mathfrak{g}$ is defined as a hom-Lie algebra $T^{*} \mathfrak{g}=\left(\mathfrak{g} \oplus \mathfrak{g}^{*},[., .]_{\mathfrak{g} \oplus \mathfrak{g}^{*}}, \phi_{\mathfrak{g}} \oplus \phi_{\mathfrak{g}^{*}}\right)$ endowed with the symplectic form $\omega$ given by (4.1), where $\phi_{\mathfrak{g}^{*}}=\left(\phi_{\mathfrak{g}}\right)^{*}$.

A representation of a hom-Lie algebra $\left(\mathfrak{g},[\cdot, \cdot], \phi_{\mathfrak{g}}\right)$ is a triple $(V, A, \rho)$ where $V$ is a vector space, $A \in g l(V)$ and $\rho: \mathfrak{g} \rightarrow g l(V)$ is a linear map satisfying

$$
\left\{\begin{array}{l}
\rho\left(\phi_{\mathfrak{g}}(u)\right) \circ A=A \circ \rho(u), \\
\rho([u, v]) \circ A=\rho\left(\phi_{\mathfrak{g}}(u)\right) \circ \rho(v)-\rho\left(\phi_{\mathfrak{g}}(v)\right) \circ \rho(u),
\end{array}\right.
$$

for any $u, v \in \mathfrak{g}$. If we consider $V^{*}$ as the dual vector space of $V$ then we can define a linear map $\rho^{*}: \mathfrak{g} \rightarrow g l\left(V^{*}\right)$ by

$$
\prec \rho^{*}(u)(\alpha), v \succ=-\prec \alpha, \rho(u)(v) \succ,
$$

for any $u \in \mathfrak{g}, v \in V, \alpha \in V^{*}$, where $\prec \rho^{*}(u)(\alpha), v \succ$ is defined by $\rho^{*}(u)(\alpha)(v)$. A representation $(V, A, \rho)$ is called admissible if $\left(V^{*}, A^{*}, \rho^{*}\right)$ is also a representation of $\mathfrak{g}$ in which $A^{*}$ is the transpose of the endomorphism $A$. In [14], it is shown that the representation $(V, A, \rho)$ is admissible if and only if the following conditions are satisfied

$$
\left\{\begin{array}{l}
A \circ \rho\left(\phi_{\mathfrak{g}}(u)\right)=\rho(u) \circ A \\
A \circ \rho([u, v])=\rho(u) \circ \rho\left(\phi_{\mathfrak{g}}(v)\right)-\rho(v) \circ \rho\left(\phi_{\mathfrak{g}}(u)\right) .
\end{array}\right.
$$

Let $\left(\mathfrak{g},[\cdot, \cdot], \phi_{\mathfrak{g}}\right)$ be a hom-Lie algebra and $a d: \mathfrak{g} \rightarrow \operatorname{End}(\mathfrak{g})$ be an operator defined for any $u, v \in \mathfrak{g}$ by $\operatorname{ad}(u)(v)=[u, v]$. Moreover, it is easy to see that

$$
a d[u, v] \circ \phi_{\mathfrak{g}}=a d\left(\phi_{\mathfrak{g}}(u)\right) \circ a d(v)-a d\left(\phi_{\mathfrak{g}}(v)\right) \circ \operatorname{ad}(u) .
$$

Thus $\left(\mathfrak{g}, \phi_{\mathfrak{g}}, a d\right)$ is a representation of $\mathfrak{g}$, which is called the adjoint representation of $\mathfrak{g}$ (see [14], for more details). For an involutive hom-Lie algebra $\left(\mathfrak{g},[\cdot, \cdot], \phi_{\mathfrak{g}}\right)$, the adjoint representation $\left(\mathfrak{g}, \phi_{\mathfrak{g}}, a d\right)$ is admissible [12].

A hom-algebra $\left(V, \cdot, \phi_{V}\right)$ is a hom-left-symmetric algebra if the following identity holds

$$
a s s_{\phi_{V}}(u, v, w)=a s s_{\phi_{V}}(v, u, w),
$$


where

$$
a s s_{\phi_{V}}(u, v, w)=(u \cdot v) \cdot \phi_{V}(w)-\phi_{V}(u) \cdot(v \cdot w),
$$

for any $u, v, w \in V$. Note that setting $[u, v]=u \cdot v-v \cdot u$, we conclude that $\left(V,[\cdot, \cdot], \phi_{V}\right)$ is a hom-Lie algebra.

Proposition 4.3. Let $\left(V, \cdot, \phi_{V}\right)$ be a hom-left symmetric algebra and for any $u \in V, L_{u}$ be the left multiplication operator on $u \in V$ (i.e., $L_{u} v=u \cdot v$ for any $\left.v \in V\right)$. Then $\left(V, \phi_{V}, L\right)$ is a representation of the hom-Lie algebra $V$, where $V$ is equipped with the induced hom-Lie bracket and $L: V \rightarrow g l(V)$ with $u \rightarrow L_{u}$.

Proof. We must show that

$$
\left\{\begin{array}{l}
L_{\phi_{V}(u)} \circ \phi_{V}=\phi_{V} \circ L_{u} \\
L_{[u, v]} \circ \phi_{V}=L_{\phi_{V}(u)} \circ L_{v}-L_{\phi_{V}(v)} \circ L_{u}
\end{array}\right.
$$

If we consider the hom-left-symmetric algebra $V$, then for any $u, v, w \in V$ we have

$$
(u \cdot v) \cdot \phi_{V}(w)-\phi_{V}(u) \cdot(v \cdot w)=(v \cdot u) \cdot \phi_{V}(w)-\phi_{V}(v) \cdot(u \cdot w) .
$$

Setting $u \cdot v-v \cdot u=[u, v]$ and $L_{u} v=u \cdot v$, in the above equation we get

$$
L_{[u, v]} \circ \phi_{V}=L_{\phi_{V}(u)} \circ L_{v}-L_{\phi_{V}(v)} \circ L_{u} .
$$

Moreover, we have $\phi_{V}(u \cdot v)=\phi_{V}(u) \cdot \phi_{V}(v)$, that is $L_{\phi_{V}(u)} \phi_{V}(v)=\phi_{V}\left(L_{u} v\right)$. Thus we have (4.4).

Corollary 4.4. Let $\left(V, \cdot, \phi_{V}\right)$ be an involutive hom-left symmetric algebra. Then the representation $\left(V, \phi_{V}, L\right)$ is admissible, i.e., $\left(V^{*}, \phi_{V^{*}}^{*}, L^{*}\right)$ is a representation of $V$. In particular, $\phi_{V^{*}}^{*}$ is involutive.

Proof. According to (4.3), it is sufficient to prove

$$
\left\{\begin{array}{l}
\phi_{V} \circ L_{\phi_{V}(u)}=L_{u} \circ \phi_{V}, \\
\phi_{V} \circ L_{[u, v]}=L_{u} \circ L_{\phi_{V}(v)}-L_{v} \circ L_{\phi_{V}(u)} .
\end{array}\right.
$$

If $u, v \in V$, then using $\phi_{V}(u \cdot v)=\phi_{V}(u) \cdot \phi_{V}(v)$ we obtain $\phi_{V}\left(\phi_{V}(u) \cdot v\right)=u \cdot \phi_{V}(v)$. Considering $\phi_{V}(u) \cdot v=L_{\phi_{V}(u)} v$, we deduce $\phi_{V}\left(L_{\phi_{V}(u)} v\right)=L_{u} \phi_{V}(v)$. On the other hand, for any $w \in V$ we have

$$
(u \cdot v) \cdot \phi_{V}(w)-(v \cdot u) \cdot \phi_{V}(w)=\phi_{V}(u) \cdot(v \cdot w)-\phi_{V}(v) \cdot(u \cdot w) .
$$

Contracting the above equation with $\phi_{V}$ implies that

$$
\phi_{V}\left((u \cdot v) \cdot \phi_{V}(w)-(v \cdot u) \cdot \phi_{V}(w)\right)=u \cdot \phi_{V}(v \cdot w)-v \cdot \phi_{V}(u \cdot w) .
$$

Setting $L_{u} v=u \cdot v$ in the above equation yields

$$
\phi_{V}\left(L_{[u, v]} \phi_{V}(w)\right)=L_{u} L_{\phi_{V}(v)} \phi_{V}(w)-L_{v} L_{\phi_{V}(u)} \phi_{V}(w) .
$$

Therefore we have the assertion.

Proposition 4.5. Let $\left(V, \cdot, \phi_{V}\right)$ be an involutive hom-left-symmetric algebra and $\left(V, \phi_{V}, L\right)$ be a representation of the hom-Lie algebra $V$. Then $\left(V \oplus V^{*}, \cdot, \Phi\right)$ is a hom-left symmetric algebra where and $\Phi$ are given by

$$
\left\{\begin{array}{c}
\left(u, a^{*}\right) \cdot\left(v, b^{*}\right):=\left(u \cdot v, L_{\phi_{V}(u)}^{*} b^{*}\right), \\
\Phi\left(u, a^{*}\right):=\left(\phi_{V}(u), \phi_{V^{*}}^{*}\left(a^{*}\right)\right),
\end{array}\right.
$$

for any $u, v \in V, a^{*}, b^{*} \in V^{*}$. 
Proof. Using (4.5), we have

$$
\begin{aligned}
& \Phi\left(\left(u, a^{*}\right) \cdot\left(v, b^{*}\right)\right)=\Phi\left(u \cdot v, L_{\phi_{V}(u)}^{*} b^{*}\right)=\left(\phi_{V}(u \cdot v), \phi_{V^{*}}^{*}\left(L_{\phi_{V}(u)}^{*} b^{*}\right)\right) \\
& =\left(\phi_{V}(u) \cdot \phi_{V}(v), \phi_{V^{*}}^{*}\left(L_{\phi_{V}(u)}^{*} b^{*}\right)\right) \text {, }
\end{aligned}
$$

and so

$$
\Phi\left(u, a^{*}\right) \cdot \Phi\left(v, b^{*}\right)=\left(\phi_{V}(u), \phi_{V^{*}}^{*}\left(a^{*}\right)\right) \cdot\left(\phi_{V}(v), \phi_{V^{*}}^{*}\left(b^{*}\right)\right)=\left(\phi_{V}(u) \cdot \phi_{V}(v), L_{u}^{*} \phi_{V^{*}}^{*}\left(b^{*}\right)\right) .
$$

But $L$ is an admissible representation on $V$, i.e., $\phi_{V^{*}}^{*}\left(L_{\phi_{V}(u)}^{*} b^{*}\right)=L_{u}^{*} \phi_{V^{*}}^{*}\left(b^{*}\right)$. Thus

$$
\Phi\left(\left(u, a^{*}\right) \cdot\left(v, b^{*}\right)\right)=\Phi\left(u, a^{*}\right) \cdot \Phi\left(v, b^{*}\right),
$$

which implies that $\Phi$ is a hom-algebra structure on $V \oplus V^{*}$. Now, we show that the product given by (4.5) is hom-left symmetric. A direct computation yields

$$
\begin{aligned}
& \left(\left(u, a^{*}\right) \cdot\left(v, b^{*}\right)\right) \cdot \Phi\left(w, c^{*}\right)-\Phi\left(u, a^{*}\right) \cdot\left(\left(v, b^{*}\right) \cdot\left(w, c^{*}\right)\right)-\left(\left(v, b^{*}\right) \cdot\left(u, a^{*}\right)\right) \cdot \Phi\left(w, c^{*}\right) \\
& +\Phi\left(v, b^{*}\right) \cdot\left(\left(u, a^{*}\right) \cdot\left(w, c^{*}\right)\right) \\
& =\left(L_{[u, v]} \phi_{V}(w)-L_{\phi_{V}(u)} L_{v} w+L_{\phi_{V}(v)} L_{u} w, L_{\phi_{V}([u, v])}^{*} \phi_{V^{*}}^{*}\left(c^{*}\right)-L_{u}^{*} L_{\phi_{V}(v)}^{*} c^{*}+L_{v}^{*} L_{\phi_{V}(u)}^{*} c^{*}\right) .
\end{aligned}
$$

On the other hand, we have

$$
\begin{gathered}
L_{[u, v]} \phi_{V}(w)=L_{\phi_{V}(u)} L_{v} w-L_{\phi_{V}(v)} L_{u} w, \\
L_{[u, v]}^{*} \phi_{V^{*}}^{*}\left(c^{*}\right)=L_{\phi_{V}(u)}^{*} L_{v}^{*} c^{*}-L_{\phi_{V}(v)}^{*} L_{u}^{*} c^{*} .
\end{gathered}
$$

Putting $u:=\phi_{V}(u), v:=\phi_{V}(v)$ in (4.7) we deduce

$$
L_{\phi_{V}([u, v])}^{*} \phi_{V^{*}}^{*}\left(c^{*}\right)=L_{u}^{*} L_{\phi_{V}(v)}^{*} c^{*}-L_{v}^{*} L_{\phi_{V}(u)}^{*} c^{*} .
$$

Setting the above equations in (4.6) we conclude the assertion.

A hom-algebra $\left(V, \cdot, \phi_{V}\right)$ is called hom-Lie-admissible algebra if its commutator bracket that is given by $[u, v]=u \cdot v-v \cdot u$, for any $u, v \in V$ satisfies the hom-Jacobi identity.

Proposition 4.6. The hom-left symmetric algebra $\left(V \oplus V^{*}, \cdot, \Phi\right)$ is an involutive hom-Lie algebra with commutator bracket, where $\cdot$ and $\Phi$ are given by (4.5).

Proof. First, we show that $\left(V \oplus V^{*}, \cdot, \Phi\right)$ is a hom-Lie-admissible algebra. If we set

$$
\left[\left(u, a^{*}\right),\left(v, b^{*}\right)\right]=\left(u, a^{*}\right) \cdot\left(v, b^{*}\right)-\left(v, b^{*}\right) \cdot\left(u, a^{*}\right), \quad \forall u, v \in V, \quad \forall a^{*}, b^{*} \in V^{*},
$$

then using (4.5) we have

$$
\left[\left(u, a^{*}\right),\left(v, b^{*}\right)\right]=\left(u \cdot v-v \cdot u, L_{\phi_{V}(u)}^{*} b^{*}-L_{\phi_{V}(v)}^{*} a^{*}\right) .
$$

Obviously, we have

$$
\left[\left(u, a^{*}\right),\left(v, b^{*}\right)\right]=-\left[\left(v, b^{*}\right),\left(u, a^{*}\right)\right] .
$$

Now, we check the hom-Jacobi identity. Applying (4.8), we get

$$
\begin{aligned}
& \circlearrowleft_{\left(u, a^{*}\right),\left(v, b^{*}\right),\left(w, c^{*}\right)}\left[\Phi\left(u, a^{*}\right),\left[\left(v, b^{*}\right),\left(w, c^{*}\right)\right]\right] \\
& =\circlearrowleft_{\left(u, a^{*}\right),\left(v, b^{*}\right),\left(w, c^{*}\right)}\left[\left(\phi_{V}(u), \phi_{V^{*}}^{*}\left(a^{*}\right)\right),\left(v \cdot w-w \cdot v, L_{\phi_{V}(v)}^{*} c^{*}-L_{\phi_{V}(w)}^{*} b^{*}\right)\right] \\
& =\circlearrowleft_{\left(u, a^{*}\right),\left(v, b^{*}\right),\left(w, c^{*}\right)}\left(\phi_{V}(u) \cdot(v \cdot w-w \cdot v)-(v \cdot w-w \cdot v) \cdot \phi_{V}(u),\right. \\
& L_{u}^{*}\left(L_{\phi_{V}(v)}^{*} c^{*}-L_{\phi_{V}(w)}^{*} b^{*}\right) \\
& \left.-L_{\phi_{V}([v, w])}^{*} \phi_{V^{*}}^{*}\left(a^{*}\right)\right) .
\end{aligned}
$$

Since $V$ is a hom-left-symmetric algebra, then

$$
(u \cdot v) \cdot \phi_{V}(w)-\phi_{V}(u) \cdot(v \cdot w)=(v \cdot u) \cdot \phi_{V}(w)-\phi_{V}(v) \cdot(u \cdot w) .
$$

On the other hand, $\left(V^{*}, \phi_{V^{*}}^{*}, L^{*}\right)$ is a representation of $V$. Therefore

$$
L_{\phi_{V}([v, w])}^{*} \circ \phi_{V^{*}}^{*}=L_{v}^{*} \circ L_{\phi_{V}(w)}^{*}-L_{w}^{*} \circ L_{\phi_{V}(v)}^{*} .
$$


The above equations imply

$$
\begin{aligned}
& \circlearrowleft\left(u, a^{*}\right),\left(v, b^{*}\right),\left(w, c^{*}\right)\left[\Phi\left(u, a^{*}\right),\left[\left(v, b^{*}\right),\left(w, c^{*}\right)\right]\right] \\
& =\left(\phi_{V}(u) \cdot(v \cdot w-w \cdot v)-(v \cdot w-w \cdot v) \cdot \phi_{V}(u)+\phi_{V}(v) \cdot(w \cdot u-u \cdot w)\right. \\
& -(w \cdot u-u \cdot w) \cdot \phi_{V}(v)+\phi_{V}(w) \cdot(u \cdot v-v \cdot u)-(u \cdot v-v \cdot u) \cdot \phi_{V}(w), L_{u}^{*}\left(L_{\phi_{V}(v)}^{*} c^{*}\right. \\
& \left.-L_{\phi_{V}(w)}^{*} b^{*}\right)-\left(L_{v}^{*} \circ L_{\phi_{V}(w)}^{*}-L_{w}^{*} \circ L_{\phi_{V}(v)}^{*}\right)\left(a^{*}\right)+L_{v}^{*}\left(L_{\phi_{V}(w)}^{*} a^{*}-L_{\phi_{V}(u)}^{*} c^{*}\right) \\
& -\left(L_{w}^{*} \circ L_{\phi_{V}(u)}^{*}-L_{u}^{*} \circ L_{\phi_{V}(w)}^{*}\right)\left(b^{*}\right)+L_{w}^{*}\left(L_{\phi_{V}(u)}^{*} b^{*}-L_{\phi_{V}(v)}^{*} a^{*}\right) \\
& -\left(L_{u}^{*} \circ L_{\phi_{V}(v)}^{*}-L_{v}^{*} \circ L_{\phi_{V}(u)}^{*}\right)\left(c^{*}\right)=0,
\end{aligned}
$$

which conclude the hom-Jacobi identity. Using (4.5) we obtain

$$
\begin{aligned}
\Phi\left[\left(u, a^{*}\right),\left(v, b^{*}\right)\right] & =\Phi\left(u \cdot v-v \cdot u, L_{\phi_{V}(u)}^{*} b^{*}-L_{\phi_{V}(v)}^{*} a^{*}\right) \\
& =\left(\phi_{V}(u \cdot v-v \cdot u), \phi_{V^{*}}^{*}\left(L_{\phi_{V}(u)}^{*} b^{*}-L_{\phi_{V}(v)}^{*} a^{*}\right)\right) \\
& =\left(\phi_{V}(u) \cdot \phi_{V}(v)-\phi_{V}(v) \cdot \phi_{V}(u), L_{u}^{*} \phi_{V^{*}}^{*}\left(b^{*}\right)-L_{v}^{*} \phi_{V^{*}}^{*}\left(a^{*}\right)\right) \\
& =\left[\left(\phi_{V}(u), \phi_{V^{*}}^{*}\left(a^{*}\right)\right),\left(\phi_{V}(v), \phi_{V^{*}}^{*}\left(b^{*}\right)\right)\right]=\left[\Phi\left(u, a^{*}\right), \Phi\left(v, b^{*}\right)\right] .
\end{aligned}
$$

Thus $\left(V \oplus V^{*}, \cdot, \Phi\right)$ is a hom-Lie algebra with commutator bracket. Finally, we get

$$
\Phi^{2}\left(u, a^{*}\right)=\Phi\left(\phi_{V}(u), \phi_{V^{*}}^{*}\left(a^{*}\right)\right)=\left(\phi_{V}^{2}(u),\left(\phi_{V^{*}}^{*}\right)^{2}\left(a^{*}\right)\right)=\left(u, a^{*}\right),
$$

i.e., $\Phi$ is involutive.

Proposition 4.7. Let $\left(V, \cdot, \phi_{V}\right)$ be an involutive hom-left-symmetric algebra and $\left(V, \phi_{V}, L\right)$ be a representation of the hom-Lie algebra $V$. Then

$$
\prec L_{\phi_{V}(u)}^{*} a^{*}, \phi_{V}(v) \succ=-\prec u \cdot v, \phi_{V^{*}}^{*}\left(a^{*}\right) \succ .
$$

Proof. We have

$$
\begin{aligned}
\prec \phi_{V^{*}}^{*}\left(a^{*}\right), u \cdot v \succ & =\prec a^{*}, \phi_{V}(u \cdot v) \succ=\prec a^{*}, \phi_{V}(u) \cdot \phi_{V}(v) \succ=\prec a^{*}, L_{\phi_{V}(u)} \phi_{V}(v) \succ \\
& =-\prec L_{\phi_{V}(u)}^{*} a^{*}, \phi_{V}(v) \succ,
\end{aligned}
$$

for any $u, v \in V$ and $a^{*} \in V^{*}$.

Theorem 4.8. Let $\left(V \oplus V^{*},[\cdot, \cdot], \Phi\right)$ be a hom-Lie algebra under the assumptions of Proposition 4.6. If we consider a non-degenerate bilinear form $\Omega_{V \oplus V^{*}}$ on $V \oplus V^{*}$ as follows

$$
\Omega_{V \oplus V^{*}}\left(\left(u, a^{*}\right),\left(v, b^{*}\right)\right)=\prec b^{*}, u \succ-\prec a^{*}, v \succ, \quad \forall u, v \in V, \quad \forall a^{*}, b^{*} \in V^{*},
$$

then $V \oplus V^{*}$ is a phase space.

Proof. Using (4.5) and (4.10), we get

$$
\begin{aligned}
\Omega_{V \oplus V^{*}}\left(\Phi\left(u, a^{*}\right), \Phi\left(v, b^{*}\right)\right) & =\Omega_{V \oplus V^{*}}\left(\left(\phi_{V}(u), \phi_{V^{*}}^{*}\left(a^{*}\right)\right),\left(\phi_{V}(v), \phi_{V^{*}}^{*}\left(b^{*}\right)\right)\right) \\
& =\prec \phi_{V^{*}}\left(b^{*}\right), \phi_{V}(u) \succ-\prec \phi_{V^{*}}^{*}\left(a^{*}\right), \phi_{V}(v) \succ \\
& =\prec b^{*}, u \succ-\prec a^{*}, v \succ=\Omega_{V \oplus V^{*}}\left(u+a^{*}, v+b^{*}\right) .
\end{aligned}
$$

Also, (4.9) implies

$$
\begin{aligned}
& \circlearrowleft_{\left(u, a^{*}\right),\left(v, b^{*}\right),\left(w, c^{*}\right)} \Omega_{V \oplus V^{*}}\left(\left[\left(u, a^{*}\right),\left(v, b^{*}\right)\right], \Phi\left(\left(w, c^{*}\right)\right)\right) \\
& =\circlearrowleft_{\left(u, a^{*}\right),\left(v, b^{*}\right),\left(w, c^{*}\right)} \Omega_{V \oplus V^{*}}\left(u \cdot v-v \cdot u, L_{\phi_{V}(u)}^{*} b^{*}-L_{\phi_{V}(v)}^{*} a^{*},\left(\phi_{V}(w), \phi_{V^{*}}^{*}\left(c^{*}\right)\right)\right) \\
& =\prec \phi_{V^{*}}^{*}\left(c^{*}\right), u \cdot v-v \cdot u \succ-\prec L_{\phi_{V}(u)}^{*} b^{*}-L_{\phi_{V}(v)}^{*} a^{*}, \phi_{V}(w) \succ+\prec \phi_{V^{*}}^{*}\left(a^{*}\right), v \cdot w-w \cdot v \succ \\
& -\prec L_{\phi_{V}(v)}^{*} c^{*}-L_{\phi_{V}(w)}^{*} b^{*}, \phi_{V}(u) \succ+\prec \phi_{V^{*}}^{*}\left(b^{*}\right), w \cdot u-u \cdot w \succ \\
& -\prec L_{\phi_{V}(w)}^{*} a^{*}-L_{\phi_{V}(u)}^{*} c^{*}, \phi_{V}(v) \succ=0 .
\end{aligned}
$$

Thus $\Omega_{V \oplus V^{*}}$ is a 2-hom-cocycle form and consequently $V \oplus V^{*}$ is a phase space. 
Lemma 4.9. Let $\left(V, \cdot, \phi_{V}\right)$ be an involutive hom-left symmetric algebra. Then for any representation $\left(V, \phi_{V}, L\right)$ we have

$$
\phi_{V^{*}}^{*}\left(u^{*}\right)=\left(\phi_{V}(u)\right)^{*} .
$$

Proof. Let $\langle\cdot, \cdot\rangle$ be a pseudo-Riemannian metric on $V$. For any $v \in V$, we have

$$
\phi_{V^{*}}^{*}\left(u^{*}\right)(v)=u^{*}\left(\phi_{V}(v)\right)=\left\langle u, \phi_{V}(v)\right\rangle,
$$

and

$$
\left(\phi_{V}(u)\right)^{*}(v)=\left\langle\phi_{V}(u), v\right\rangle .
$$

Since $\phi_{V}$ is involutive, then (2.1) implies that the above equations are equal.

Proposition 4.10. Let $\left(V \oplus V^{*},[\cdot, \cdot], \Phi\right)$ be a hom-Lie algebra under the assumptions of Proposition 4.6. Then the linear map $\mathrm{f}: V \oplus V^{*} \rightarrow V \oplus V^{*}$ defined by

$$
\mathcal{J}\left(u, a^{*}\right)=\left(-\phi_{V}(a), \phi_{V^{*}}^{*}\left(u^{*}\right)\right), \quad \forall u \in V, \forall a^{*} \in V^{*},
$$

is a complex structure on the hom-Lie algebra $V \oplus V^{*}$.

Proof. Applying Lemma 4.9, we obtain

$$
\begin{aligned}
\mathcal{J}^{2}\left(u, a^{*}\right) & =\mathcal{J}\left(-\phi_{V}(a), \phi_{V^{*}}^{*}\left(u^{*}\right)\right)=\mathcal{J}\left(-\phi_{V}(a),\left(\phi_{V}(u)\right)^{*}\right)=\left(-\phi_{V}\left(\phi_{V}(u)\right),-\phi_{V^{*}}^{*}\left(\phi_{V}(a)\right)^{*}\right) \\
& =-\left(u, a^{*}\right) .
\end{aligned}
$$

Also $\Phi \circ \mathcal{J}=\mathcal{J} \circ \Phi$, because

$$
\begin{aligned}
& (\Phi \circ \mathcal{J})\left(u, a^{*}\right)=\Phi\left(-\phi_{V}(a), \phi_{V^{*}}^{*}\left(u^{*}\right)\right)=\left(-\phi_{V}^{2}(a),\left(\phi_{V^{*}}^{*}\right)^{2}\left(u^{*}\right)\right)=\left(-a, u^{*}\right), \\
& (\mathcal{J} \circ \Phi)\left(u, a^{*}\right)=\mathcal{J}\left(\phi_{V}(u), \phi_{V^{*}}^{*}\left(a^{*}\right)\right)=\left(-\phi_{V}\left(\phi_{V}(a)\right), \phi_{V^{*}}^{*}\left(\phi_{V}(u)\right)^{*}\right)=\left(-a, u^{*}\right) .
\end{aligned}
$$

Thus $\mathcal{J}$ is an almost complex structure on the hom-Lie algebra $V \oplus V^{*}$. Using (4.5) we have

$$
\begin{aligned}
& \mathcal{N}\left(\left(u, a^{*}\right),\left(v, b^{*}\right)\right)=\left[(\Phi \circ \mathcal{J})\left(u, a^{*}\right),(\Phi \circ \mathcal{J})\left(v, b^{*}\right)\right]-(\Phi \circ \mathcal{J})\left[(\Phi \circ \mathcal{J})\left(u, a^{*}\right),\left(v, b^{*}\right)\right] \\
& -(\Phi \circ \mathcal{J})\left[\left(u, a^{*}\right),(\Phi \circ \mathcal{J})\left(v, b^{*}\right)\right]-\left[\left(u, a^{*}\right),\left(v, b^{*}\right)\right] \\
& =\left[\left(-a, u^{*}\right),\left(-b, v^{*}\right)\right]-(\Phi \circ \mathcal{J})\left[\left(-a, u^{*}\right),\left(v, b^{*}\right)\right]-(\Phi \circ \mathcal{J})\left[\left(u, a^{*}\right),\left(-b, v^{*}\right)\right]-\left[\left(u, a^{*}\right),\left(v, b^{*}\right)\right] \\
& =\left(a \cdot b-b \cdot a,-L_{\phi_{V}(a)}^{*} v^{*}+L_{\phi_{V}(b)}^{*} u^{*}\right)-(\Phi \circ \mathcal{J})\left(-a \cdot v+v \cdot a,-L_{\phi_{V}(a)}^{*} b^{*}-L_{\phi_{V}(v)}^{*} u^{*}\right) \\
& -(\Phi \circ \mathcal{J})\left(-u \cdot b+b \cdot u, L_{\phi_{V}(u)}^{*} v^{*}+L_{\phi_{V}(b)}^{*} a^{*}\right)-\left(u \cdot v-v \cdot u, L_{\phi_{V}(u)}^{*} b^{*}-L_{\phi_{V}(v)}^{*} a^{*}\right) .
\end{aligned}
$$

Now, let $u \in \mathfrak{g}$. Then using (4.2) we have

$$
\phi_{V^{*}}^{*}\left(L_{\phi_{V}(a)}^{*} v^{*}\right)(u)=L_{\phi_{V}(a)}^{*} v^{*}\left(\phi_{V}(u)\right)=-v^{*}\left(L_{\phi_{V}(a)} \phi_{V}(u)\right)=-\left\langle v, \phi_{V}(a) \cdot \phi_{V}(u)\right\rangle .
$$

Applying (2.4) in the above equation we conclude

$$
\phi_{V^{*}}^{*}\left(L_{\phi_{V}(a)}^{*} v^{*}\right)(u)=\left\langle\phi_{V}(a) \cdot \phi_{V}(v), u\right\rangle=\left\langle L_{\phi_{V}(a)} \phi_{V}(v), u\right\rangle=\left(L_{\phi_{V}(a)} \phi_{V}(v)\right)^{*}(u),
$$

i.e.,

$$
\phi_{V^{*}}^{*}\left(L_{\phi_{V}(a)}^{*} v^{*}\right)=\left(L_{\phi_{V}(a)} \phi_{V}(v)\right)^{*} .
$$

The last equation and $(\Phi \circ \mathcal{J})\left(u, a^{*}\right)=\left(-a, u^{*}\right)$ imply

$$
\begin{aligned}
(\Phi \circ \mathcal{J})\left(a \cdot v, L_{\phi_{V}(a)}^{*} b^{*}\right) & =(\mathcal{J} \circ \Phi)\left(a \cdot v, L_{\phi_{V}(a)}^{*} b^{*}\right)=\mathcal{J}\left(\phi_{V}(a) \cdot \phi_{V}(v), \phi_{V^{*}}^{*}\left(L_{\phi_{V}(a)}^{*} b^{*}\right)\right) \\
& =\mathcal{J}\left(L_{\phi_{V}(a)} \phi_{V}(v), \phi_{V^{*}}^{*}\left(L_{\phi_{V}(a)}^{*} b^{*}\right)\right) \\
& =\mathcal{J}\left(\left(\phi_{V^{*}}^{*}\left(L_{\phi_{V}(a)}^{*} v^{*}\right)\right)^{*},\left(L_{\phi_{V}(a)} \phi_{V}(b)\right)^{*}\right) \\
& =\left(-\phi_{V}\left(L_{\phi_{V}(a)} \phi_{V}(b)\right), \phi^{*}\left(\phi_{V^{*}}^{*}\left(L_{\phi_{V}(a)}^{*} v^{*}\right)\right)\right)=\left(-a \cdot b, L_{\phi_{V}(a)}^{*} v^{*}\right) .
\end{aligned}
$$


Finally the above equation and (4.12) yield

$$
\begin{aligned}
\mathcal{N}\left(\left(u, a^{*}\right),\left(v, b^{*}\right)\right)=( & a \cdot b-b \cdot a-u \cdot v+v \cdot u-a \cdot b-v \cdot u+u \cdot v+b \cdot a,-L_{\phi_{V}(a)}^{*} v^{*} \\
& +L_{\phi_{V}(b)}^{*} u^{*}-L_{\phi_{V}(u)}^{*} b^{*}+L_{\phi_{V}(v)}^{*} a^{*}+L_{\phi_{V}(a)}^{*} v^{*}-L_{\phi_{V}(v)}^{*} a^{*}-L_{\phi_{V}(b)}^{*} u^{*} \\
& \left.+L_{\phi_{V}(u)}^{*} b^{*}\right)=0,
\end{aligned}
$$

which gives $\mathcal{J}$ is a complex structure on the hom-Lie algebra $V \oplus V^{*}$.

\section{Kähler hom-Lie algebras}

In this section, we introduce Kähler structures on hom-Lie algebras and we present an example of these structures. Also, we show that there does not exist non-abelian Hermitian and Kähler proper hom-Lie algebras of dimension 2. In particular, we classify non-abelian Hermitian and Kähler hom-Lie algebras of dimension 2.

\subsection{Kähler structures on hom-Lie algebras}

Definition 5.1. A Kähler hom-Lie algebra is a pseudo-Riemannian hom-Lie algebra $\left(\mathfrak{g},[\cdot, \cdot], \phi_{\mathfrak{g}},\langle\cdot, \cdot\rangle\right)$ endowed with an almost complex structure $J$, such that $\phi_{\mathfrak{g}} \circ J$ is invariant with respect to the hom-Levi-Civita product, i.e., $L_{u} \circ \phi_{\mathfrak{g}} \circ J=\phi_{\mathfrak{g}} \circ J \circ L_{u}$ for any $u \in \mathfrak{g}$.

Note that condition $\left(u \cdot\left(\phi_{\mathfrak{g}} \circ J\right)\right)(v)=\left(\phi_{\mathfrak{g}} \circ J\right)(u \cdot v)$ is equivalent with

$$
\left(\phi_{\mathfrak{g}} \circ J\right)(u) \cdot\left(\phi_{\mathfrak{g}} \circ J\right)(v)=\left(\phi_{\mathfrak{g}} \circ J\right)\left(\left(\phi_{\mathfrak{g}} \circ J\right)(u) \cdot v\right),
$$

and

$$
u \cdot v=-\left(\phi_{\mathfrak{g}} \circ J\right)\left(u \cdot\left(\phi_{\mathfrak{g}} \circ J\right)(v)\right) .
$$

Remark 5.2. Using (3.1), (5.1) and (5.2) we obtain

$$
\begin{aligned}
N(u, v)= & \left(\phi_{\mathfrak{g}} \circ J\right)(u) \cdot\left(\phi_{\mathfrak{g}} \circ J\right)(v)-\left(\phi_{\mathfrak{g}} \circ J\right)(v) \cdot\left(\phi_{\mathfrak{g}} \circ J\right)(u)-\left(\phi_{\mathfrak{g}} \circ J\right)\left(\left(\phi_{\mathfrak{g}} \circ J\right)(u) \cdot v\right. \\
& \left.-v \cdot\left(\phi_{\mathfrak{g}} \circ J\right)(u)\right)-\left(\phi_{\mathfrak{g}} \circ J\right)\left(u \cdot\left(\phi_{\mathfrak{g}} \circ J\right)(v)-\left(\phi_{\mathfrak{g}} \circ J\right)(v) \cdot u\right)-u \cdot v-v \cdot u=0 .
\end{aligned}
$$

So, the almost structure $J$ introduced in Definition 5.1 is integrable.

Example 5.3. We consider a 4-dimensional linear space $\mathfrak{g}$ with an arbitrary basis $\left\{e_{1}, e_{2}, e_{3}, e_{4}\right\}$. We define the bracket and linear map $\phi_{\mathfrak{g}}$ on $\mathfrak{g}$ as follows

$$
\left[e_{1}, e_{2}\right]=-a e_{3}, \quad\left[e_{1}, e_{3}\right]=b e_{2}, \quad\left[e_{2}, e_{4}\right]=-a e_{2}, \quad\left[e_{3}, e_{4}\right]=a e_{3},
$$

and

$$
\phi_{\mathfrak{g}}\left(e_{1}\right)=-e_{1}, \quad \phi_{\mathfrak{g}}\left(e_{2}\right)=e_{2}, \quad \phi_{\mathfrak{g}}\left(e_{3}\right)=-e_{3}, \quad \phi_{\mathfrak{g}}\left(e_{4}\right)=e_{4} .
$$

The above bracket is not a Lie bracket on $\mathfrak{g}$ if $a \neq 0$ and $b \neq 0$, because

$$
\left[e_{1},\left[e_{3}, e_{4}\right]\right]+\left[e_{3},\left[e_{4}, e_{1}\right]+\left[e_{4},\left[e_{1}, e_{3}\right]\right]=\left[e_{1}, a e_{3}\right]+\left[e_{4}, b e_{2}\right]=2 a b e_{2} .\right.
$$

It is easy to see that

$$
\begin{array}{ll}
{\left[\phi_{\mathfrak{g}}\left(e_{1}\right), \phi_{\mathfrak{g}}\left(e_{2}\right)\right]=a e_{3}=\phi_{\mathfrak{g}}\left(\left[e_{1}, e_{2}\right]\right),} & {\left[\phi_{\mathfrak{g}}\left(e_{1}\right), \phi_{\mathfrak{g}}\left(e_{3}\right)\right]=b e_{2}=\phi_{\mathfrak{g}}\left(\left[e_{1}, e_{3}\right]\right),} \\
{\left[\phi_{\mathfrak{g}}\left(e_{2}\right), \phi_{\mathfrak{g}}\left(e_{4}\right)\right]=-a e_{2}=\phi_{\mathfrak{g}}\left(\left[e_{2}, e_{4}\right]\right),} & {\left[\phi_{\mathfrak{g}}\left(e_{3}\right), \phi_{\mathfrak{g}}\left(e_{4}\right)\right]=-a e_{3}=\phi_{\mathfrak{g}}\left(\left[e_{3}, e_{4}\right]\right),}
\end{array}
$$

i.e., $\phi_{\mathfrak{g}}$ is the algebra morphism. Also, we can deduce

$$
\left[\phi_{\mathfrak{g}}\left(e_{i}\right),\left[e_{j}, e_{k}\right]\right]+\left[\phi_{\mathfrak{g}}\left(e_{j}\right),\left[e_{k}, e_{i}\right]+\left[\phi_{\mathfrak{g}}\left(e_{k}\right),\left[e_{i}, e_{j}\right]\right]=0, \quad i, j, k=1,2,3,4 .\right.
$$

Thus $\left(\mathfrak{g},[\cdot, \cdot], \phi_{\mathfrak{g}}\right)$ is a hom-Lie algebra. Now we consider the bilinear skew-symmetric nondegenerate form $\Omega$ as follows:

$$
\left[\begin{array}{cccc}
0 & 0 & -A & 0 \\
0 & 0 & 0 & \frac{a}{b} A \\
A & 0 & 0 & 0 \\
0 & -\frac{a}{b} A & 0 & 0
\end{array}\right] .
$$


Then we get

$$
\begin{array}{ll}
\Omega\left(\phi_{\mathfrak{g}}\left(e_{1}\right), \phi_{\mathfrak{g}}\left(e_{3}\right)\right)=-A=\Omega\left(e_{1}, e_{3}\right), & \Omega\left(\phi_{\mathfrak{g}}\left(e_{2}\right), \phi_{\mathfrak{g}}\left(e_{4}\right)\right)=\frac{a}{b} A=\Omega\left(e_{2}, e_{4}\right), \\
\Omega\left(\phi_{\mathfrak{g}}\left(e_{1}\right), \phi_{\mathfrak{g}}\left(e_{2}\right)\right)=0=\Omega\left(e_{1}, e_{2}\right), & \Omega\left(\phi_{\mathfrak{g}}\left(e_{1}\right), \phi_{\mathfrak{g}}\left(e_{4}\right)\right)=0=\Omega\left(e_{1}, e_{4}\right), \\
\Omega\left(\phi_{\mathfrak{g}}\left(e_{2}\right), \phi_{\mathfrak{g}}\left(e_{3}\right)\right)=0=\Omega\left(e_{2}, e_{3}\right), & \Omega\left(\phi_{\mathfrak{g}}\left(e_{3}\right), \phi_{\mathfrak{g}}\left(e_{4}\right)\right)=0=\Omega\left(e_{3}, e_{4}\right),
\end{array}
$$

and

$$
\Omega\left(\left[e_{i}, e_{j}\right], \phi_{\mathfrak{g}}\left(e_{k}\right)\right)+\Omega\left(\left[e_{j}, e_{k}\right], \phi_{\mathfrak{g}}\left(e_{i}\right)\right)+\Omega\left(\left[e_{k}, e_{i}\right], \phi_{\mathfrak{g}}\left(e_{j}\right)\right)=0, \quad i, j, k=1,2,3,4 .
$$

The above relations show that $\Omega$ is 2 -hom-cocycle and so $\left(\mathfrak{g},[\cdot, \cdot], \phi_{\mathfrak{g}}, \Omega\right)$ is a symplectic hom-Lie algebra. We define a metric on $\mathfrak{g}$ as follows

$$
\left[\begin{array}{cccc}
A & 0 & 0 & 0 \\
0 & \frac{a}{b} A & 0 & 0 \\
0 & 0 & A & 0 \\
0 & 0 & 0 & \frac{a}{b} A
\end{array}\right] .
$$

We can easily deduce that $\left\langle\phi\left(e_{i}\right), e_{j}\right\rangle=0=\left\langle e_{i}, \phi\left(e_{j}\right)\right\rangle$, for all $i, j=1,2,3,4$, except

$$
\begin{array}{rlrl}
\left\langle\phi_{\mathfrak{g}}\left(e_{1}\right), e_{1}\right\rangle & =-A=\left\langle e_{1}, \phi_{\mathfrak{g}}\left(e_{1}\right)\right\rangle, & \left\langle\phi_{\mathfrak{g}}\left(e_{2}\right), e_{2}\right\rangle & =\frac{a}{b} A=\left\langle e_{2}, \phi_{\mathfrak{g}}\left(e_{2}\right)\right\rangle, \\
\left\langle\phi_{\mathfrak{g}}\left(e_{3}\right), e_{3}\right\rangle & =-A=\left\langle e_{3}, \phi_{\mathfrak{g}}\left(e_{3}\right)\right\rangle, & \left\langle\phi_{\mathfrak{g}}\left(e_{4}\right), e_{4}\right\rangle=\frac{a}{b} A=\left\langle e_{4}, \phi_{\mathfrak{g}}\left(e_{4}\right)\right\rangle .
\end{array}
$$

Thus $(2.3)$ holds and so $\left(\mathfrak{g},[\cdot, \cdot], \phi_{\mathfrak{g}},\langle\cdot, \cdot\rangle\right)$ is a pseudo-Riemannian hom-Lie algebra. If isomorphism $J$ is determined as

$$
J\left(e_{1}\right)=e_{3}, \quad J\left(e_{2}\right)=e_{4}, \quad J\left(e_{3}\right)=-e_{1}, \quad J\left(e_{4}\right)=-e_{2},
$$

then using the above equations, it follows that

$$
J^{2}\left(e_{i}\right)=-\phi_{\mathfrak{g}}^{2}\left(e_{i}\right)=-e_{i}, \quad i=1,2,3,4,
$$

and

$$
\begin{array}{ll}
\left(J \circ \phi_{\mathfrak{g}}\right) e_{1}=-e_{3}=\left(\phi_{\mathfrak{g}} \circ J\right) e_{1}, & \left(J \circ \phi_{\mathfrak{g}}\right) e_{2}=e_{4}=\left(\phi_{\mathfrak{g}} \circ J\right) e_{2}, \\
\left(J \circ \phi_{\mathfrak{g}}\right) e_{3}=e_{1}=\left(\phi_{\mathfrak{g}} \circ J\right) e_{3}, & \left(J \circ \phi_{\mathfrak{g}}\right) e_{4}=-e_{2}=\left(\phi_{\mathfrak{g}} \circ J\right) e_{4} .
\end{array}
$$

Thus $J$ is an almost complex structure on $\left(g,[\cdot, \cdot], \phi_{\mathfrak{g}}\right)$. Also, a simple calculation shows that $N\left(e_{i}, e_{j}\right)=0$, for all $i, j=1,2,3,4$, i.e., $J$ is a complex structure on $\left(\mathfrak{g},[\cdot, \cdot], \phi_{\mathfrak{g}}\right)$. It is not hard to find that

$$
\left\langle\left(\phi_{\mathfrak{g}} \circ J\right)\left(e_{i}\right),\left(\phi_{\mathfrak{g}} \circ J\right)\left(e_{j}\right)>=<e_{i}, e_{j}\right\rangle, \quad \forall i, j=1,2,3,4,
$$

and hence $\left(\mathfrak{g},[\cdot, \cdot], \phi_{\mathfrak{g}},\langle\cdot, \cdot\rangle, J\right)$ is a Hermitian hom-Lie algebra. Now, we study the Kählerian property for this hom-Lie algebra. At first we must obtain the hom-Levi-Civita product for it. If we denote this product with $\cdot$, then we have $e_{i} \cdot e_{j}=\sum_{k=1}^{4} A_{i j}^{k} e_{k}$, for all $i, j=1,2,3,4$. From Koszul's formula given by $(2.2)$ we get $\left\langle e_{1} \cdot e_{1}, \phi\left(e_{i}\right)\right\rangle=0$, for all $i=1,2,3,4$, which give $e_{1} \cdot e_{1}=0$. Again (2.2) gives $\left\langle e_{1} \cdot e_{2}, \phi_{\mathfrak{g}}\left(e_{i}\right)\right\rangle=0$, for all $i=1,2,3,4$, which leads to $A_{12}^{1}=A_{12}^{2}=A_{12}^{3}=A_{12}^{4}=0$. Therefore we deduce $e_{1} \cdot e_{2}=0$. But $\left[e_{1}, e_{2}\right]=e_{1} \cdot e_{2}-e_{2} \cdot e_{1}$ implies $e_{2} \cdot e_{1}=a e_{3}$. In a similar way, it can be verified that

$$
\begin{gathered}
e_{3} \cdot e_{1}=-b e_{2}, \quad e_{2} \cdot e_{2}=a e_{4}, \quad e_{2} \cdot e_{4}=-a e_{2}, \quad e_{3} \cdot e_{4}=a e_{3}, e_{3} \cdot e_{3}=b e_{4}, \\
e_{2} \cdot e_{3}=e_{3} \cdot e_{2}=-a e_{1},
\end{gathered}
$$

and

$$
e_{1} \cdot e_{3}=e_{1} \cdot e_{4}=e_{4} \cdot e_{1}=e_{4} \cdot e_{2}=e_{4} \cdot e_{3}=e_{4} \cdot e_{4}=0 .
$$

It can be checked easily that the hom-Levi-Civita product computed above satisfies in (5.1). Thus $\left(\mathfrak{g},[\cdot, \cdot], \phi_{\mathfrak{g}}, J,\langle\cdot, \cdot\rangle\right)$ is a Kähler hom-Lie algebra. 
Proposition 5.4. Let $\left(\mathfrak{g},[\cdot, \cdot], \phi_{\mathfrak{g}},\langle\cdot, \cdot\rangle, J\right)$ be a Kähler hom-Lie algebra. Then $\left(\mathfrak{g},[\cdot, \cdot], \phi_{\mathfrak{g}}, \Omega\right)$ is a symplectic hom-Lie algebra, where

$$
\Omega(u, v)=\left\langle\left(\phi_{\mathfrak{g}} \circ J\right) u, v\right\rangle .
$$

Proof. Applying (5.3) and (2.3) we obtain

$$
\begin{aligned}
\Omega( & {\left.[u, v], \phi_{\mathfrak{g}}(w)\right)+\Omega\left([v, w], \phi_{\mathfrak{g}}(u)\right)+\Omega\left([w, u], \phi_{\mathfrak{g}}(v)\right) } \\
= & -\left\langle[u, v],\left(\phi_{\mathfrak{g}} \circ J\right)\left(\phi_{\mathfrak{g}}(w)\right)\right\rangle-\left\langle[v, w],\left(\phi_{\mathfrak{g}} \circ J\right)\left(\phi_{\mathfrak{g}}(u)\right)\right\rangle-\left\langle[w, u],\left(\phi_{\mathfrak{g}} \circ J\right)\left(\phi_{\mathfrak{g}}(v)\right)\right\rangle \\
= & -\left\langle u \cdot v,\left(\phi_{\mathfrak{g}} \circ J\right)\left(\phi_{\mathfrak{g}}(w)\right)\right\rangle+\left\langle v \cdot u,\left(\phi_{\mathfrak{g}} \circ J\right)\left(\phi_{\mathfrak{g}}(w)\right)\right\rangle-\left\langle v \cdot w,\left(\phi_{\mathfrak{g}} \circ J\right)\left(\phi_{\mathfrak{g}}(u)\right)\right\rangle \\
& +\left\langle w \cdot v,\left(\phi_{\mathfrak{g}} \circ J\right)\left(\phi_{\mathfrak{g}}(u)\right)\right\rangle-\left\langle w \cdot u,\left(\phi_{\mathfrak{g}} \circ J\right)\left(\phi_{\mathfrak{g}}(v)\right)\right\rangle+\left\langle u \cdot w,\left(\phi_{\mathfrak{g}} \circ J\right)\left(\phi_{\mathfrak{g}}(v)\right)\right\rangle,
\end{aligned}
$$

for any $u, v, w \in \mathfrak{g}$. But using (2.4) and (5.2) we conclude

$$
\begin{aligned}
\left\langle u \cdot v,\left(\phi_{\mathfrak{g}} \circ J\right)\left(\phi_{\mathfrak{g}}(w)\right)\right\rangle= & -\left\langle\left(\phi_{\mathfrak{g}} \circ J\right)\left(u \cdot\left(\phi_{\mathfrak{g}} \circ J\right)(v)\right),\left(\phi_{\mathfrak{g}} \circ J\right)\left(\phi_{\mathfrak{g}}(w)\right)\right\rangle \\
& \left.=-\left\langle u \cdot\left(\phi_{\mathfrak{g}} \circ J\right) v,\left(\phi_{\mathfrak{g}} w\right)\right\rangle=\wedge u \cdot w, \phi_{\mathfrak{g}}\left(\phi_{\mathfrak{g}} \circ J\right)(v)\right\rangle .
\end{aligned}
$$

Setting the above equation in (5.4) we get

$$
\begin{aligned}
& \Omega\left([u, v], \phi_{\mathfrak{g}}(w)\right)+\Omega\left([v, w], \phi_{\mathfrak{g}}(u)\right)+\Omega\left([w, u], \phi_{\mathfrak{g}}(v)\right) \\
& =-\left\langle u \cdot w,\left(\phi_{\mathfrak{g}}{ }^{2} \circ J\right) v\right\rangle+\left\langle v \cdot w,\left(\phi_{\mathfrak{g}}{ }^{2} \circ J\right) u\right\rangle-\langle v \cdot w, J u\rangle \\
& \quad+\left\langle w \cdot u,\left(\phi_{\mathfrak{g}}{ }^{2} \circ J\right) v\right\rangle-\langle w \cdot u, J v\rangle+\langle u \cdot w, J v\rangle \\
& =0 .
\end{aligned}
$$

Moreover (5.3) implies

$$
\Omega\left(\phi_{\mathfrak{g}}(u), \phi_{\mathfrak{g}}(v)\right)=\left\langle\left(\phi_{\mathfrak{g}} \circ J\right) \phi_{\mathfrak{g}}(u), \phi_{\mathfrak{g}}(v)\right\rangle=\left\langle J(u), \phi_{\mathfrak{g}}(v)\right\rangle=\left\langle\left(\phi_{\mathfrak{g}} \circ J\right) u, v\right\rangle=\Omega(u, v) .
$$

Theorem 5.5. Let $\left(\mathfrak{g},[\cdot, \cdot], \phi_{\mathfrak{g}}, \omega\right)$ be an involutive symplectic hom-Lie algebra. Then there exists a hom-left-symmetric algebra structure a on $\mathfrak{g}$ satisfying

$$
\omega\left(\mathbf{a}(u, v), \phi_{\mathfrak{g}}(w)\right)=-\omega\left(\phi_{\mathfrak{g}}(v),[u, w]\right),
$$

such that

$$
\mathbf{a}(u, v)-\mathbf{a}(v, u)=[u, v],
$$

for any $u, v \in \mathfrak{g}$.

Proof. See [12], for more details.

According to the above theorem, a Kähler hom-Lie algebra has two products, the homLevi-Civita product and the hom-left symmetric product $\mathbf{a}$ associated with $\left(\mathfrak{g},[\cdot, \cdot], \phi_{\mathfrak{g}}, \Omega\right)$.

According the above notions and Propositions 4.5-4.10 we deduce the following:

Corollary 5.6. If $\left(\mathfrak{g},[\cdot, \cdot], \phi_{\mathfrak{g}},\langle\cdot, \cdot\rangle, J, \Omega\right)$ is a Kähler hom-Lie algebra, then $\left(\mathfrak{g}, \mathbf{a}, \phi_{\mathfrak{g}}\right)$ is a hom-left symmetric algebra. Also, $\left(\mathfrak{g} \oplus \mathfrak{g}^{*}, \mathbf{a}, \Phi\right)$ is a hom-left symmetric algebra, where $\mathfrak{g}^{*}$ is the dual space of $\mathfrak{g}$ and $\mathbf{a}$ and $\Phi$ are given by (4.5). Moreover, $\left(\mathfrak{g} \oplus \mathfrak{g}^{*}, \Omega_{\mathfrak{g} \oplus \mathfrak{g}^{*}}\right)$ is a phase space of hom-Lie algebra $\mathfrak{g}$ where $\Omega_{\mathfrak{g} \oplus \mathfrak{g}^{*}}$ is given by (4.10).

Corollary 5.7. Let $\left(\mathfrak{g},[\cdot, \cdot], \phi_{\mathfrak{g}},\langle\cdot, \cdot\rangle, J, \Omega\right)$ be a Kähler hom-Lie algebra. Then there is a complex structure $\mathcal{J}$ on $\mathfrak{g} \oplus \mathfrak{g}^{*}$ given by (4.11). 


\subsection{Kähler structures on non-abelian hom-Lie algebras of dimension 2}

In [13], the same authors determined all non-abelian (involutive) hom-Lie algebras of dimension 2 and non-abelian pseudo-Riemannian hom-Lie algebra of dimension 2 as follows:

All non-abelian hom-Lie algebras of dimension 2 are as $\left(\mathfrak{g},[\cdot, \cdot], \phi_{\mathfrak{g}}\right)$ with

$$
\left(\phi_{\mathfrak{g}}\left(e_{1}\right)=e_{1}+B e_{2}, \quad \phi_{\mathfrak{g}}\left(e_{2}\right)=C e_{2}, \quad C \neq 0\right) \quad \text { or } \quad\left(\phi_{\mathfrak{g}}\left(e_{1}\right)=A e_{1}+B e_{2}, \quad \phi_{\mathfrak{g}}\left(e_{2}\right)=0\right),
$$

where $\left\{e_{1}, e_{2}\right\}$ is a basis of $\mathfrak{g}$ such that $\left[e_{1}, e_{2}\right]=e_{2}$.

Also, all non-abelian involutive hom-Lie algebras of dimension 2 are as $\left(\mathfrak{g},[\cdot, \cdot], \widehat{\phi}_{\mathfrak{g}}\right)$, $\left(\mathfrak{g},[\cdot, \cdot], \bar{\phi}_{\mathfrak{g}}\right)$ and $\left(\mathfrak{g},[\cdot, \cdot], \widetilde{\phi}_{\mathfrak{g}}\right)$, where

$$
\begin{aligned}
& \left(\widehat{\phi}_{\mathfrak{g}}\left(e_{1}\right)=e_{1}, \widehat{\phi}_{\mathfrak{g}}\left(e_{2}\right)=e_{2}\right), \quad\left(\bar{\phi}_{\mathfrak{g}}\left(e_{1}\right)=e_{1}, \bar{\phi}_{\mathfrak{g}}\left(e_{2}\right)=-e_{2}\right), \\
& \left(\widetilde{\phi}_{\mathfrak{g}}\left(e_{1}\right)=e_{1}+B e_{2}, \widetilde{\phi}_{\mathfrak{g}}\left(e_{2}\right)=-e_{2}, \quad B \neq 0\right) .
\end{aligned}
$$

In the sequel, we will consider involutive hom-Lie algebras of dimension 2.

Moreover, all non-abelian pseudo-Riemannian hom-Lie algebra of dimension 2 are as

$$
\left(\mathfrak{g},[\cdot, \cdot], \widehat{\phi}_{\mathfrak{g}},\langle\cdot, \cdot\rangle\right), \quad\left(\mathfrak{g},[\cdot, \cdot], \bar{\phi}_{\mathfrak{g}}, \prec \cdot, \cdot \succ\right), \quad\left(\mathfrak{g},[\cdot, \cdot], \widetilde{\phi}_{\mathfrak{g}}, \ll \cdot, \cdot \gg\right),
$$

where $\widehat{\phi}_{\mathfrak{g}}, \bar{\phi}_{\mathfrak{g}}$ and $\widetilde{\phi}_{\mathfrak{g}}$ are given by $(5.5)$ and $\langle\cdot, \cdot\rangle$ is an arbitrary bilinear symmetric nondegenerate form and $\prec \cdot, \succ \succ, \ll \cdot, \cdot \gg$ are bilinear symmetric non-degenerate forms which have the following matrix presentations:

$$
\begin{aligned}
{[\langle\cdot, \cdot\rangle]=} & {\left[\begin{array}{cc}
\left\langle e_{1}, e_{1}\right\rangle & \left\langle e_{1}, e_{2}\right\rangle \\
\left\langle e_{1}, e_{2}\right\rangle & \left\langle e_{2}, e_{2}\right\rangle
\end{array}\right], \quad\left\langle e_{1}, e_{1}\right\rangle\left\langle e_{2}, e_{2}\right\rangle-\left\langle e_{1}, e_{2}\right\rangle^{2} \neq 0, } \\
{[\prec \cdot, \cdot \succ]=} & {\left[\begin{array}{cc}
\prec e_{1}, e_{1} \succ & 0 \\
0 & \prec e_{2}, e_{2} \succ
\end{array}\right], \quad \prec e_{1}, e_{1} \succ \neq 0, \prec e_{2}, e_{2} \succ \neq 0, } \\
{[\ll \cdot, \cdot \gg]=} & {\left[\begin{array}{cc}
\ll e_{1}, e_{1} \gg & -\frac{B}{2} \ll e_{2}, e_{2} \gg \\
-\frac{B}{2} \ll e_{2}, e_{2} \gg & \ll e_{2}, e_{2} \gg
\end{array}\right], } \\
& \ll e_{1}, e_{1} \gg \ll e_{2}, e_{2} \gg-\frac{B^{2}}{4} \ll e_{2}, e_{2} \gg^{2} \neq 0 .
\end{aligned}
$$

Proposition 5.8. All non-abelian almost complex hom-Lie algebras of dimension 2 are as $\left(\mathfrak{g},[\cdot, \cdot], \widehat{\phi}_{\mathfrak{g}}, \widehat{J}\right)$, where $\widehat{\phi}_{\mathfrak{g}}$, is given by (5.5) and $\widehat{J}$ is as follows:

$$
[\widehat{J}]=\left[\begin{array}{cc}
a & b \\
c & -a
\end{array}\right], \quad a^{2}+b c=-1,
$$

and non-abelian hom-Lie algebras $\left(\mathfrak{g},[\cdot, \cdot], \bar{\phi}_{\mathfrak{g}}\right)$ and $\left(\mathfrak{g},[\cdot, \cdot], \widetilde{\phi}_{\mathfrak{g}}\right)$, do not admit any almost complex structure.

Proof. Let $\left(\mathfrak{g},[\cdot, \cdot], \phi_{\mathfrak{g}}, J\right)$ be an almost complex hom-Lie algebra of dimension 2 , such that $J=\left[\begin{array}{cc}\rho_{1}^{1} & \rho_{1}^{2} \\ \rho_{2}^{1} & \rho_{2}^{2}\end{array}\right]$. Since $J^{2}\left(e_{1}\right)=-e_{1}$ and $J^{2}\left(e_{2}\right)=-e_{2}$, thus we have

$$
\left(\rho_{1}^{1}\right)^{2}+\rho_{1}^{2} \rho_{2}^{1}=-1, \quad \rho_{1}^{2}\left(\rho_{1}^{1}+\rho_{2}^{2}\right)=0, \quad \rho_{2}^{1}\left(\rho_{1}^{1}+\rho_{2}^{2}\right)=0, \quad\left(\rho_{2}^{2}\right)^{2}+\rho_{1}^{2} \rho_{2}^{1}=-1 .
$$

Now, we consider possible cases for the above equations

Case 1. $\rho_{2}^{2}=-\rho_{1}^{1}$.

In this case, using the first and the fourth equations of (5.6) we deduce $\left(\rho_{1}^{1}\right)^{2}+\rho_{1}^{2} \rho_{2}^{1}=-1$.

Case 2. $\rho_{2}^{2} \neq-\rho_{1}^{1}$.

In this case, the second and the third equations of (5.6) imply $\rho_{1}^{2}=\rho_{2}^{1}=0$ and $\left(\rho_{1}^{1}\right)^{2}=$ 
$\left(\rho_{2}^{2}\right)^{2}=-1$. But this is not possible.

Therefore $J$ has the following matrix presentation:

$$
\left[\begin{array}{cc}
a & b \\
c & -a
\end{array}\right], \quad a^{2}+b c=-1
$$

Considering $\widehat{\phi}_{\mathfrak{g}}=I d_{\mathfrak{g}}$, then we conclude $\left(\mathfrak{g},[\cdot, \cdot], \widehat{\phi}_{\mathfrak{g}}, \widehat{J}\right)$ is an almost complex (hom-)Lie algebra where $\widehat{J}$ is given by (5.7). If we consider $\bar{\phi}_{\mathfrak{g}}$ and the matrix presentation of $\bar{J}$ is as (5.7), then condition $\left(\bar{J} \circ \bar{\phi}_{\mathfrak{g}}\right)\left(e_{1}\right)=\left(\bar{\phi}_{\mathfrak{g}} \circ \bar{J}\right)\left(e_{1}\right)$ gives $b=0$ and $\left(\bar{J} \circ \bar{\phi}_{\mathfrak{g}}\right)\left(e_{2}\right)=\left(\bar{\phi}_{\mathfrak{g}} \circ \bar{J}\right)\left(e_{2}\right)$ yields $c=0$. Consequently, we get the contradiction $a^{2}=-1$ and so $\left(\mathfrak{g},[\cdot, \cdot], \bar{\phi}_{\mathfrak{g}}, \bar{J}\right)$ cannot be an almost complex hom-Lie algebra. Finally, if we consider $\widetilde{\phi}_{\mathfrak{g}}$ and the matrix presentation of $\widetilde{J}$ is $(5.7)$, then condition $\left(\widetilde{J} \circ \widetilde{\phi}_{\mathfrak{g}}\right)\left(e_{2}\right)=\left(\widetilde{\phi}_{\mathfrak{g}} \circ \widetilde{J}\right)\left(e_{2}\right)$ yields $c=0$ and consequently, $a^{2}=-1$. So, $\widetilde{J}$ cannot be an almost complex structure on $\left(\mathfrak{g},[\cdot, \cdot], \widetilde{\phi}_{\mathfrak{g}}\right)$.

Proposition 5.9. All non-abelian almost Hermitian hom-Lie algebras of dimension 2 are as $\left(\mathfrak{g},[\cdot, \cdot], \widehat{\phi}_{\mathfrak{g}}, \widehat{J}_{i},\langle\cdot, \cdot\rangle_{i}\right), i=1,2$, where $\widehat{\phi}_{\mathfrak{g}}$ is given by (5.5) and $\widehat{J}_{i}$ and $\langle\cdot, \cdot\rangle_{i}$ have the following matrix presentations:

$$
\begin{gathered}
{\left[\widehat{J}_{1}\right]=\left[\begin{array}{cc}
a & d \\
h & -a
\end{array}\right], \quad\left[\widehat{J}_{2}\right]=\left[\begin{array}{cc}
0 & d \\
-\frac{1}{d} & 0
\end{array}\right],} \\
{\left[\langle\cdot, \cdot\rangle_{1}\right]=\left[\begin{array}{cc}
\left\langle e_{1}, e_{1}\right\rangle_{1} & -\frac{a}{d}\left\langle e_{1}, e_{1}\right\rangle_{1} \\
-\frac{a}{d}\left\langle e_{1}, e_{1}\right\rangle_{1} & -\frac{h}{d}\left\langle e_{1}, e_{1}\right\rangle_{1}
\end{array}\right], \quad\left[\langle\cdot, \cdot\rangle_{2}\right]=\left[\begin{array}{cc}
\left\langle e_{1}, e_{1}\right\rangle_{2} & 0 \\
0 & \frac{1}{d^{2}}\left\langle e_{1}, e_{1}\right\rangle_{2}
\end{array}\right],}
\end{gathered}
$$

where $a, d, h \neq 0, a^{2}+d h=-1$.

Proof. Since $\left(\mathfrak{g},[\cdot, \cdot], \widehat{\phi}_{\mathfrak{g}}\right)$ admits the almost complex structure $\widehat{J}$, i.e., $[\widehat{J}]=\left[\begin{array}{cc}a & d \\ h & -a\end{array}\right]$, then we can consider the following cases

Case 1. $a \neq 0$.

In this case, condition $\left\langle\left(\widehat{J} \circ \widehat{\phi}_{\mathfrak{g}}\right)\left(e_{1}\right),\left(\widehat{J} \circ \widehat{\phi}_{\mathfrak{g}}\right)\left(e_{1}\right)\right\rangle=\left\langle e_{1}, e_{1}\right\rangle$ gives

$$
\left\langle e_{1}, e_{2}\right\rangle=\frac{1-a^{2}}{2 a d}\left\langle e_{1}, e_{1}\right\rangle-\frac{d}{2 a}\left\langle e_{2}, e_{2}\right\rangle \text {. }
$$

Also, the condition $\left\langle\left(\widehat{J} \circ \widehat{\phi}_{\mathfrak{g}}\right)\left(e_{2}\right),\left(\widehat{J} \circ \widehat{\phi}_{\mathfrak{g}}\right)\left(e_{2}\right)\right\rangle=\left\langle e_{2}, e_{2}\right\rangle$ implies

$$
\left\langle e_{1}, e_{2}\right\rangle=\frac{h}{2 a}\left\langle e_{1}, e_{1}\right\rangle+\frac{a^{2}-1}{2 a h}\left\langle e_{2}, e_{2}\right\rangle \text {. }
$$

Moreover, $\left\langle(\widehat{J} \circ \widehat{\phi})\left(e_{1}\right),\left(\widehat{J} \circ \widehat{\phi}_{\mathfrak{g}}\right)\left(e_{2}\right)\right\rangle=\left\langle e_{1}, e_{2}\right\rangle$ yields

$$
\left\langle e_{1}, e_{2}\right\rangle=-\frac{a}{2 d}\left\langle e_{1}, e_{1}\right\rangle+\frac{a}{2 h}\left\langle e_{2}, e_{2}\right\rangle .
$$

From (5.8) and (5.9) we get

$$
\left\langle e_{2}, e_{2}\right\rangle=-\frac{h}{d}\left\langle e_{1}, e_{1}\right\rangle
$$

Setting (5.11) in (5.10), we conclude $\left\langle e_{1}, e_{2}\right\rangle=-\frac{a}{d}\left\langle e_{1}, e_{1}\right\rangle$. We denote these structures in the assertion with index 1 in what follows.

Case 2. $a=0$.

In the case, we have $h d=-1$ and hence $[\widehat{J}]=\left[\begin{array}{cc}0 & d \\ -\frac{1}{d} & 0\end{array}\right]$. In a similar way of the previous case, we obtain $\left\langle e_{2}, e_{2}\right\rangle=\frac{1}{d^{2}}\left\langle e_{1}, e_{1}\right\rangle$ and $\left\langle e_{1}, e_{2}\right\rangle=0$. We denote these structures in the assertion with index 2 in the below.

As can bee seen in Proposition 5.9, $\widehat{\phi}_{\mathfrak{g}}$ given by (5.5) is the only morphism that $\left(\mathfrak{g},[\cdot, \cdot], \widehat{\phi}_{\mathfrak{g}}, \widehat{J}_{i},\langle\cdot, \cdot\rangle_{i}\right), i=1,2$, are non-abelian almost Hermitian hom-Lie algebras. Since $\widehat{\phi}_{\mathfrak{g}}=I d_{\mathfrak{g}}$, so we conclude the following: 
Corollary 5.10. There does not exists a non-abelian almost Hermitian proper hom-Lie algebra of dimension 2 .

From above corollary, we deduce the following

Corollary 5.11. There exists no non-abelian Kähler proper hom-Lie algebra of dimension 2 .

Proposition 5.12 ([13]). The hom-Levi-Civita product on the pseudo-Riemannian homLie algebra $\left(\mathfrak{g},[\cdot, \cdot], \widehat{\phi}_{\mathfrak{g}},\langle\cdot, \cdot\rangle\right)$ is

$$
e_{1} \cdot e_{1}=0, \quad e_{1} \cdot e_{2}=0, \quad e_{2} \cdot e_{1}=-e_{2}, \quad e_{2} \cdot e_{2}=\frac{\left\langle e_{2}, e_{2}\right\rangle}{\left\langle e_{1}, e_{1}\right\rangle} e_{1},
$$

if $\left\langle e_{1}, e_{2}\right\rangle=0$, and

$$
\begin{aligned}
& e_{1} \cdot e_{1}=\frac{\left\langle e_{1}, e_{2}\right\rangle^{2}}{\operatorname{det}[\langle\cdot, \cdot\rangle]} e_{1}-\frac{\left\langle e_{1}, e_{1}\right\rangle\left\langle e_{1}, e_{2}\right\rangle}{\operatorname{det}[\langle\cdot, \cdot\rangle]} e_{2}, \\
& e_{1} \cdot e_{2}=\frac{\left\langle e_{2}, e_{2}\right\rangle}{\operatorname{det}[\langle\cdot, \cdot\rangle]} e_{1}-\frac{\left\langle e_{1}, e_{2}\right\rangle}{\operatorname{det}[\langle\cdot, \cdot\rangle]} e_{2}, \\
& e_{2} \cdot e_{1}=\frac{\left\langle e_{2}, e_{2}\right\rangle}{\operatorname{det}[\langle\cdot, \cdot\rangle]} e_{1}-\frac{\operatorname{det}[\langle\cdot, \cdot\rangle]+\left\langle e_{1}, e_{2}\right\rangle}{\operatorname{det}[\langle\cdot, \cdot\rangle]} e_{2}, \\
& e_{2} \cdot e_{2}=\frac{\left\langle e_{2}, e_{2}\right\rangle^{2}}{\operatorname{det}[\langle\cdot, \cdot\rangle]\left\langle e_{1}, e_{2}\right\rangle} e_{1}-\frac{\left\langle e_{2}, e_{2}\right\rangle}{\operatorname{det}[\langle\cdot, \cdot\rangle]} e_{2},
\end{aligned}
$$

if $\left\langle e_{1}, e_{2}>\neq 0\right.$.

Proposition 5.13. All non-abelian Kähler hom-Lie algebras of dimension 2 are as $\left(\mathfrak{g},[\cdot, \cdot], \widehat{\phi}_{\mathfrak{g}}, \widehat{J}_{i},\langle\cdot, \cdot\rangle_{i}\right), i=1,2$, where $\widehat{\phi}_{\mathfrak{g}}$ is given by (5.5) and $\widehat{J}_{i}$ and $<,>_{i}$ have the following matrix presentations:

$$
\begin{aligned}
& \left\{\begin{array}{l}
{\left[\widehat{J}_{1}\right]=\left[\begin{array}{cc}
a & d \\
h & -a
\end{array}\right], \quad\left[\langle\cdot, \cdot\rangle_{1}\right]=\left[\begin{array}{cc}
-\frac{d}{a} & 1 \\
1 & \frac{h}{a}
\end{array}\right], \quad e_{1} \cdot e_{1}=a^{2} e_{1}+a d e_{2},} \\
e_{1} \cdot e_{2}=a h e_{1}-a^{2} e_{2}, \quad e_{2} \cdot e_{1}=a h e_{1}-\left(a^{2}+1\right) e_{2}, \quad e_{2} \cdot e_{2}=h^{2} e_{1}-a h e_{2},
\end{array}\right. \\
& \left\{\begin{aligned}
{\left[\widehat{J}_{2}\right] } & =\left[\begin{array}{ll}
0 & d \\
\frac{1}{d} & 0
\end{array}\right], \quad\left[\langle\cdot, \cdot\rangle_{2}\right]=\left[\begin{array}{cc}
\left\langle e_{1}, e_{1}\right\rangle_{2} & 0 \\
0 & \frac{\left\langle e_{1}, e_{1}\right\rangle_{2}}{d^{2}}
\end{array}\right], \\
e_{2} \cdot e_{1} & =-e_{2}, \quad e_{1} \cdot e_{1}=e_{1} \cdot e_{2}=0, \quad e_{2} \cdot e_{2}=\frac{1}{d^{2}} e_{1},
\end{aligned}\right.
\end{aligned}
$$

where $a, d \neq 0, a^{2}+a d=-1$.

Proof. From Proposition 5.9, it is easy to see that $N_{\widehat{J}_{1} \circ \widehat{\phi}_{\mathfrak{g}}}=N_{\widehat{J}_{2} \circ \widehat{\phi}_{\mathfrak{g}}}=0$, i.e., $\widehat{J}_{1}, \widehat{J}_{2}$ are Hermitian structures on $\mathfrak{g}$. Applying Proposition 5.12, we study compatibility of the structures determined in Proposition 5.9 and these products. We consider two cases as follows:

Case 1. $\left\langle e_{1}, e_{2}\right\rangle=0$.

In this case, since $\langle\cdot, \cdot\rangle_{1}$, is not a pseudo-Riemannian metric, when $\left\langle e_{1}, e_{2}\right\rangle=0$. Thus we consider $\langle\cdot, \cdot\rangle_{2}$. In this case, the product (5.12) becomes

$$
e_{1} \cdot e_{1}=e_{1} \cdot e_{2}=0, \quad e_{2} \cdot e_{1}=-e_{2}, \quad e_{2} \cdot e_{2}=\frac{1}{d^{2}} e_{1} .
$$

Considering the above product, we see that $(5.2)$ holds for $\left(\mathfrak{g},[],, \widehat{\phi}_{\mathfrak{g}}, \widehat{J}_{2},\langle\cdot, \cdot\rangle_{2}\right)$. Hence this structure is Kähler.

Case 2. $<e_{1}, e_{2}>\neq 0$.

In this case considering $\widehat{J}_{1}$ and $\langle\cdot, \cdot\rangle_{1}$ from Proposition 5.9, we deduce that (5.13)-(5.16) 
reduce to

$$
\begin{aligned}
e_{1} \cdot e_{1} & =\frac{a^{2}\left\langle e_{1}, e_{1}\right\rangle_{1}^{2}}{d^{2} \operatorname{det}\left[\langle\cdot, \cdot\rangle_{1}\right]} e_{1}+\frac{a\left\langle e_{1}, e_{1}\right\rangle_{1}^{2}}{d \operatorname{det}\left[\langle\cdot, \cdot\rangle_{1}\right]} e_{2}, \\
e_{1} \cdot e_{2} & =\frac{-h\left\langle e_{1}, e_{1}\right\rangle_{1}}{d \operatorname{det}\left[\langle\cdot, \cdot\rangle_{1}\right]} e_{1}+\frac{a\left\langle e_{1}, e_{1}\right\rangle_{1}}{d \operatorname{det}\left[\langle\cdot, \cdot\rangle_{1}\right]} e_{2}, \\
e_{2} \cdot e_{1} & =\frac{-h\left\langle e_{1}, e_{1}\right\rangle_{1}}{d \operatorname{det}\left[\langle\cdot, \cdot\rangle_{1}\right]} e_{1}-\frac{\operatorname{det}\left[\langle\cdot, \cdot\rangle_{1}\right]-\frac{a}{d}\left\langle e_{1}, e_{1}\right\rangle_{1}}{\operatorname{det}\left[\langle\cdot, \cdot\rangle_{1}\right]} e_{2}, \\
e_{2} \cdot e_{2} & =\frac{-h^{2}\left\langle e_{1}, e_{1}\right\rangle_{1}}{a d \operatorname{det}\left[\langle\cdot, \cdot\rangle_{1}\right]} e_{1}+\frac{h\left\langle e_{1}, e_{1}\right\rangle_{1}}{d \operatorname{det}\left[\langle\cdot, \cdot\rangle_{1}\right]} e_{2} .
\end{aligned}
$$

Using the above equations and $a^{2}+h d=-1$ we get

$$
-\widehat{J}_{1}\left(e_{1} \cdot \widehat{J}_{1}\left(e_{1}\right)\right)=\frac{a^{2}\left\langle e_{1}, e_{1}\right\rangle_{1}^{2}}{d^{2} \operatorname{det}\left[\langle\cdot, \cdot\rangle_{1}\right]} e_{1}-\frac{\left\langle e_{1}, e_{1}\right\rangle_{1}}{\operatorname{det}\left[\langle\cdot, \cdot\rangle_{1}\right]} e_{2} .
$$

As $e_{1} \cdot e_{1}=-\widehat{J}_{1}\left(e_{1} \cdot \widehat{J}_{1}\left(e_{1}\right)\right)$, thus $\left\langle e_{1}, e_{1}\right\rangle_{1}=-\frac{d}{a}$. In this case, $\operatorname{det}\left[\langle\cdot, \cdot\rangle_{1}\right]=\frac{1}{a^{2}}$ and hence $\left[\langle\cdot, \cdot\rangle_{1}\right]$ and the Levi-Civita product reduce to (5.17). Applying it we have

$$
\begin{gathered}
-\widehat{J}_{1}\left(e_{1} \cdot \widehat{J}_{1}\left(e_{2}\right)\right)=a h e_{1}-a^{2} e_{2}=e_{1} \cdot e_{2}, \\
-\widehat{J}_{1}\left(e_{2} \cdot \widehat{J}_{1}\left(e_{1}\right)\right)=a h e_{1}-\left(a^{2}+1\right) e_{2}=e_{2} \cdot e_{1}, \\
-\widehat{J}_{1}\left(e_{2} \cdot \widehat{J}_{1}\left(e_{2}\right)\right)=h^{2} e_{1}-a h e_{2}=e_{2} \cdot e_{2} .
\end{gathered}
$$

Therefore we conclude (5.2).

\section{References}

[1] A. Andrada, M.L. Barberis, and I. Dotti, Classification of abelian complex structures on 6-dimensional Lie algebras, J. London Math. Soc. 83, 232-255, 2011.

[2] A. Andrada and S. Salamon, Complex product structures on Lie algebras, Forum Math. 17, 261-295, 2005.

[3] S. Benayadi and A. Makhlouf, Hom-Lie algebras with symmetric invariant nondegenerate bilinear forms, J. Math. Phys. 76, 38-60, 2014.

[4] G. Calvaruso and A. Fino, Five-dimensional K-contact Lie algebras, Monatsh. Math. 167 (1), 35-59, 2012.

[5] J. Hartwig, D. Larsson, and S. Silvestrov, Deformations of Lie algebras using $\sigma$ derivations, J. Algebra 295, 314-361, 2006.

[6] N. Hu, q-Witt algebras, q-Lie algebras, q-holomorph structure and representations, Algebra Colloq., 6 (1), 51-70, 1999.

[7] J. Jiang, S.K. Mishra, and Y. Sheng, Hom-Lie algebras and hom-Lie groups, integration and differentiation, arXiv:1904.06515.

[8] D. Larsson and S. Silvestrov, Quasi-hom-Lie algebras, central extensions and 2cocycle-like identities, J. Algebra 288, 321-344, 2005.

[9] A. Makhlouf and S.D. Silvestrov, Hom-algebra structures, J. Gen. Lie Theory Appl. 2 (2), 51-64, 2008.

[10] A. Makhlouf and S D. Silvestrov, Notes on formal deformations of hom-associative and hom-Lie algebras, Forum Math. 22, 715-759, 2010.

[11] A. Makhlouf and D. Yau, Rota-baxter hom-lie admissible algebras, Comm. Algebra. 42, 1231-1257, 2014.

[12] E. Peyghan and L. Nourmohammadifar, Para-Kähler hom-Lie algebras, J. Algebra Appl. 18 (3), 1950044, 2019.

[13] E. Peyghan and L. Nourmohammadifar, Para-Kähler hom-Lie algebras of dimension 2, submitted. 
[14] Y. Sheng and C. Bai, A new approach to hom-Lie bialgebras, J. Algebra 399, 232-250, 2014.

[15] Y. Sheng and D. Chen, Hom-Lie 2-algebras, J. Algebra 376, 174-195, 2013.

[16] Z. Xiong, Hom-Lie groups of a class of hom-Lie algebra, arXiv:1810.07881. 